\title{
Gradyan Tabanlı Optimize Edici Algoritmasının Parametre Ayarlaması
}

\author{
Mehmet Beşkirli $^{1^{*}}$, Mehmet Fatih Tefek ${ }^{2}$ \\ $\mathbf{1}^{*}$ Şırnak Üniversitesi, Mühendislik Fakültesi, Bilgisayar Mühendisliği Bölümü, Şırnak, Türkiye, (ORCID: 0000-0002-4842-3817), mehmetbes@sirnak.edu.tr \\ ${ }^{2}$ Osmaniye Korkut Ata Üniversitesi, Mühendislik Fakültesi, Bilgisayar Mühendisliği Bölümü, Osmaniye, Türkiye, (ORCID: 0000-0003-3390-4201), \\ mehmetfatihtefek@osmaniye.edu.tr
}

(1st International Conference on Applied Engineering and Natural Sciences ICAENS 2021, November 1-3, 2021)

(DOI: 10.31590/ejosat.1010813)

ATIF/REFERENCE: Beşkirli, M. \& Tefek M.F. (2021). Gradyan Tabanlı Optimize Edici Algoritmasının Parametre Ayarlaması. Avrupa Bilim ve Teknoloji Dergisi, (28), 727-742.

$\ddot{\mathbf{O} z}$

Bu çalışmada, popülasyon tabanlı yeni bir meta-sezgisel optimizasyon algoritması olan gradyan tabanlı optimize edici (GBO) algoritmasının olasılık parametresi ayarı yapılmıştır. Gradyan tabanlı Newton yönteminden ilham alan GBO, gradyan arama kuralı (GAK) ve yerel kaçış operatörü (YKO) olmak üzere iki ana operatör kullanır. Gradyan arama kuralında, uygulanabilir alanda daha iyi arama yapmak ve daha iyi konumlar elde etmek için vektörlerin hareketi kontrol edilir. Arama eğilimini arttırmak ve GBO'nun yakınsamasını hızlandırmak amacıyla, GAK, gardyan tabanlı (GT) yöntemi konseptine dayalı olarak önerilmiştir. GAK, arama uzayında daha iyi konumlar elde etmek için keşif eğilimini geliştirmek ve yakınsama oranını hızlandırmak için gradyan tabanlı yöntemi kullanır. YKO operatörü, çözümün konumunu önemli ölçüde değiştirebilmektedir. YKO operatöründe rastgele değerlerle kıyaslanmak üzere olasılık parametresi $(p r \in(0,1))$ kullanılmaktadır. Olasılık parametresi GBO'nun çalışma performansına etkisini anlayabilmek için on iki adet tek modlu ve on iki adet çok modlu test fonksiyonları kullanılmıştır. Deneysel çalışmalarda olasılık parametre değerleri sırasıyla $0,1,0,3,0,5,0,7$ ve 0,9 olarak alınmıştır. GBO algoritmasında olasılık parametresinin önemli bir faktör olduğu ve GBO'nun çalışma performansını önemli ölçüde etkilediği belirlenmiştir. Ek olarak olasılık parametresinin 0,9 değerine yaklaştığında GBO'nun tek modlu ve çok modlu test fonksiyon sonuçlarında daha iyi değerler hesapladığı grafik ve tablolarla gösterilmiştir.

Anahtar Kelimeler: Optimizasyon, Gradyan tabanlı optimize edici, Test fonksiyonları, Algoritma parametre ayarı

\section{Parameter Adjustment of Gradient Based Optimizer Algorithm}

\begin{abstract}
In this study, the probability parameter of the gradient-based optimizer (GBO) algorithm, which is a new population-based metaheuristic optimization algorithm, is adjusted. Inspired by the gradient-based Newtonian method, GBO uses two main operators, the gradient search rule (GSR) and the local escape operator (LEO). In the gradient search rule, the movement of vectors is controlled in order to better search and obtain better positions in the applicable area. In order to increase search propensity and accelerate the convergence of GBO, GSR is proposed based on the concept of the guard-based (GT) method. GSR uses the gradient-based method to improve the exploration propensity and speed up the convergence rate to get better positions in the search space. The LEO operator can significantly change the position of the solution. In the LEO operator, the probability parameter $(\operatorname{pr} \in(0,1))$ is used to compare with random values. In order to understand the effect of probability parameter GBO on operating performance, twelve single-mode and twelve multi-mode benchmark functions are used. In experimental studies, probability parameter values were taken as $0.1,0.3,0.5,0.7$ and 0.9 , respectively. It has been determined that the probability parameter is an important factor in the GBO algorithm and it significantly affects the working performance of the GBO. In addition, graphs and tables show that GBO calculates better values in unimodal and multimodal benchmark function results when the probability parameter approaches 0.9 .
\end{abstract}

Keywords: Optimization, Gradient based optimizer, Benchmark functions, Algorithm parameter setting

*Sorumlu Yazar: mehmetbes@sirnak.edu.tr 


\section{Giriş}

Optimizsyon amaç fonksiyonunun belirli kısıtlar altında en iyi değerini elde etme işlemidir. Metasezgisel algoritmalar optimizasyon problemlerinin tümü için kesin sonuç elde edeceklerini garanti etmezler. Ancak en iyi sonucu elde etmek için uğraşırlar. Metasezgisel algoritmalar klasik yöntemlere göre daha iyi sonuç vermektedirler (Makhloufi, 2015). Bundan dolayı optimizasyon algoritmaları inşaat mühendisliği, makine mühendisliği gibi pek çok alanda kullanılmaktadır (Aala Kalananda \& Komanapalli, 2021; Beşkirli \& Dağ, 2020; Dhiman \& Kaur, 2019; Houssein, Saad, Hashim, Shaban, \& Hassaballah, 2020; Huerta et al., 2022; Kutlu Onay \& Aydemir, 2022; Salgotra, Singh, Singh, Mittal, \& Gandomi, 2021; Shabani, Asgarian, Salido, \& Asil Gharebaghi, 2020; Sulaiman, Mustaffa, Saari, \& Daniyal, 2020; Umam, Mustafid, \& Suryono, 2021). Optimizasyon algoritmaları doğadan ilham almaktadır. $\mathrm{Bu}$ sebepten optimizasyon algoritmaları biyolojik temelli, popülasyon temelli, fizik temelli, sürü temelli, sosyal temelli, gradyan tabanlı, müzik temelli ve kimya temelli tekniklerden oluşmaktadır (Beşkirli, Temurtaş, \& Özdemir, 2020). Oluşturulan algoritmalar gerçek hayatta karşılaşılan mühendislik problemlerini çözme amaçlanmıştır (Beskirli, Beskirli, Hakli, \& Uguz, 2018). Bu çalışmada ise gradya ve popülasyon tabanlı birleşiminden oluşturulan Gradyan tabanlı optimize edici (GBO) algoritması kullanılmıştır. Her ne kadar optimizasyon algoritmaları iyi sonucu elde etmek için uğraşsada algoritma üzerinde yapılacak olan iyileştirmeler ile algoritma daha iyi sonuçlar elde edebilmektedir. GBO algoritmanın en iyi parametre düzeyi bu çalışma ile araştırılmıştır. Algoritmanın parametre düzenlemesi ile elde edilecek sonucun başarısını etkilemektedir. $\mathrm{Bu}$ sebep ile literatürde yer alan birçok problem için uygun olacağını düşündüğümüz parametre değerleri tek modlu ve çok modlu test fonksiyonlarına uygulanması ile en iyi parametre değerleri tavsiye edilecektir. Bu konu ile ilgili literatürde birçok çalışma yer almaktadır. $\mathrm{Bu}$ çalışmalar incelendiğinde algoritmaların parametrelerinin en iyi değerde olduğu zaman algoritmanın performansının en üst düzeyde olduğu belirtilmiştir (Akay \& Karaboga, 2012; Alavidoost, Zarandi, Tarimoradi, \& Nemati, 2017; Beşkirli, Özdemir, \& Temurtaş, 2020; Kıran \& Find1k, 2015).

\section{Materyal ve Metot}

$\mathrm{Bu}$ çalışma için tek modlu ve çok modlu test fonksiyonları kullanılmıştır. Bunlar ile ilgili açıklamalar bu bölümde verilmiştir. Bunların yanı sıra kullanılan algoritma da bu bölümde verilmiştir.

\subsection{Test Fonksiyonları}

Tablo 1. Tek modlu fonksiyonlar

\begin{tabular}{l|l|l|c|l|c}
\hline Fn. & Adı & Arama Uzayı & Boyut & Fonksiyon & $\boldsymbol{F}_{\min }$ \\
\hline$F 1$ & Sphere & {$[-100,100]$} & 25 & $F 1(x)=\sum_{i=1}^{n} x_{i}^{2}$ & 0 \\
\hline$F 2$ & Quartic Noise & {$[-1.28,1.28]$} & 25 & $F 2(x)=\sum_{i=1}^{n} i x_{i}^{4}+\operatorname{random}[0,1]$ & 0 \\
\hline$F 3$ & Powell Sum & {$[-1,1]$} & 25 & $F 3(x)=\Sigma_{i=1}^{D}\left|x_{i}\right|^{i+1}$ & 0 \\
\hline$F 4$ & Schwefel's 2.20 & {$[-100,100]$} & 25 & $F 4(x)=\Sigma_{i=1}^{n}\left|x_{i}\right|$ & 0 \\
\hline$F 5$ & Schwefel's 2.21 & {$[-100,100]$} & 25 & $F 5(x)=\max _{i}\left(\left|x_{i}\right|, 1 \leq i \leq n\right)$ & 0 \\
\hline$F 6$ & Step & {$[-100,100]$} & 25 & $F 6(x)=\Sigma_{i=1}^{n}\left(\left[x_{i}+0.5\right]\right)^{2}$ & 0 \\
\hline$F 7$ & Stepint & {$[-5.12,5.12]$} & 25 & $\left.F 7(x)=25+\sum_{i=1}^{n}\left(\mid x_{i}\right]\right)$ & -155 \\
\hline$F 8$ & Schwefel's 1.20 & {$[-100,100]$} & 25 & $F 8(x)=\sum_{i=1}^{n}\left(\sum_{j=1}^{i} x_{j}\right)^{2}$ & 0 \\
\hline$F 9$ & Schwefel's 2.22 & {$[-100,100]$} & 25 & $F 9(x)=\sum_{i=1}^{n}\left|x_{i}\right|+\Pi_{i=1}^{n}\left|x_{i}\right|$ & 0 \\
\hline$F 10$ & Schwefel's 2.23 & {$[-10,10]$} & 25 & $F 10(x)=\sum_{i=1}^{n} x_{i}^{10}$ & 0 \\
\hline$F 11$ & Rosenbrock & {$[-30,30]$} & 25 & $F 11(x)=\sum_{i=1}^{n-1}\left[100\left(x_{i+1}-x_{i}^{2}\right)^{2}+\left(x_{i}-1\right)^{2}\right]$ & 0 \\
\hline$F 12$ & Brown & {$[-1,4]$} & 25 & $F 12(x)=\sum_{i=1}^{n-1}\left(x_{i}^{2}\right)^{\left(x_{i+1}^{2}+1\right)}+\left(x_{i+1}^{2}\right)^{x_{i}^{2}+1}$ & 0 \\
\hline
\end{tabular}

Tablo 2. Çok modlu fonksiyonlar

\begin{tabular}{l|l|l|c|l|c}
\hline Fn. & Adı & Arama Uzayı & Boyut & Fonksiyon & $\boldsymbol{F}_{\text {min }}$ \\
\hline$F 1$ & Schwefel's 2.26 & {$[-500,500]$} & 25 & $F 1(x)=418.9829 n-\sum_{i=1}^{n}-x_{i} \sin \left(\sqrt{\left|x_{i}\right|}\right)$ & 0 \\
\hline$F 2$ & Rastrigin & {$[-5.12,5.12]$} & 25 & $F 2(x)=\sum_{i=1}^{n}\left[x_{i}^{2}-10 \cos \left(2 \pi x_{i}\right)+10\right]$ & 0 \\
\hline$F 3$ & Periodic & {$[-10,10]$} & 25 & $F 3(x)=1+\sum_{i=1}^{n} \sin ^{2}\left(x_{i}\right)-0.1 e^{\left(\Sigma_{i=1}^{n} x_{i}^{2}\right)}$ & 0.9 \\
\hline$F 4$ & Qing & {$[-500,500]$} & 25 & $F 4(x)=\sum_{i=1}^{n}\left(x^{2}-i\right)^{2}$ & 0 \\
\hline$F 5$ & Alpine N. 1 & {$[-10,10]$} & 25 & $F 5(x)=\sum_{i=1}^{n}\left|x_{i} \sin \left(x_{i}\right)+0.1 x_{i}\right|$ & 0 \\
\hline$F 6$ & Xin-She Yang & {$[-5,5]$} & 25 & $F 6(x)=\sum_{i=1}^{n} \epsilon_{i}\left|x_{i}\right|^{i}$ & 0 \\
\hline
\end{tabular}


European Journal of Science and Technology

\begin{tabular}{|c|c|c|c|c|c|}
\hline F7 & Ackley & {$[-32,32]$} & 25 & $\begin{aligned} F 7(x)=-20 \exp ( & \left.-0.2 \sqrt{1 / n \sum_{i=1}^{n} x_{i}^{2}}\right) \\
& -\exp \left(1 / n \Sigma_{i=1}^{n} \cos \left(2 \pi x_{i}\right)\right)+20 \\
& +e\end{aligned}$ & 0 \\
\hline$F 8$ & Trignometric 2 & {$[-500,500]$} & 25 & $\begin{aligned} F 8(x)=\sum_{i=1}^{n} 8 \sin ^{2}[ & \left.7\left(x_{i}-0.9\right)^{2}\right] \\
& +6 \sin ^{2}\left[14\left(x_{1}-0.9\right)^{2}\right] \\
& +\left(x_{i}-0.9\right)^{2}\end{aligned}$ & 1 \\
\hline F9 & Salomon & {$[-100,100]$} & 25 & $F 9(x)=1-\cos \left(2 \pi \sqrt{\sum_{i=1}^{n} x_{i}^{2}}\right)+0.1 \sqrt{\sum_{i=1}^{n} x_{i}^{2}}$ & 0 \\
\hline$F 10$ & Styblinski-Tang & {$[-5,5]$} & 25 & $F 10(x)=\frac{1}{2} \sum_{i=1}^{n}\left(x_{i}^{4}-16 x_{i}^{2}+5 x_{i}\right)$ & -1174.98 \\
\hline$F 11$ & Griewank & {$[-100,100]$} & 25 & $F 11(x)=1 / 4000 \sum_{i=1}^{n} x_{i}^{2}-\Pi_{i=1}^{n} \cos \left(x_{i} / \sqrt{i}\right)+1$ & 0 \\
\hline$F 12$ & Xin-She Yang N.4 & {$[-10,10]$} & 25 & $F 12(x)=\left(\sum_{i=1}^{n} \sin ^{2}\left(x_{i}\right)-e^{-\Sigma_{i=1}^{n} x_{i}^{2}}\right) e^{-\sum_{i=1}^{n} \sin ^{2} \sqrt{\left|x_{i}\right|}}$ & -1 \\
\hline
\end{tabular}

\subsubsection{GBO Algoritmast}

GBO algoritması 2020 y1lında Ahmadianfar vd. tarafindan sürekli optimizasyon problemlerinin çözümü için önerilmiştir (Ahmadianfar, Bozorg-Haddad, \& Chu, 2020). Grandyan ve popülasyon tabanlı yöntemleri birleşiminden oluşan GBO algoritması arama uzayını keşfetmek için Newton yöntemini kullanmışlardır. GBO üç ana bileşenden oluşmaktadır. İlk olarak başlangıç aşamasıdır. İkincisi grandyan arama kuralıdır. Sonuncusu ise yerel kaçış operatörüdür. Başlangıç aşamasında, kontrol parametresi, yineleme sayıs1, popülasyon sayısı yer almaktadır. $\mathrm{Bu}$ algoritmada popülasyon sayısına vektör sayısı denilmektedir. D-boyutlu bir arama uzayında $\mathrm{N}$ tane vektör yer almaktadır. Bu vektör ise Denklem 1'deki gibi ifade edilmiştir.

$X_{n, d}=\left[X_{n, 1}, X_{n, 2}, \ldots, X_{n, D}\right], n=1,2, \ldots, N$

$d=1,2, \ldots, D$

Grandyan arama kuralı ise uygulanabilir alanda daha iyi arama yapmak ve daha iyi konumlar elde etmek için vektörlerin hareketi kontrol edildiği bölümdür. Arama eğilimini arttırmak ve yakınsama hızını arttırmak için kullanılmaktadır. Grandyan arama kuralının hesaplaması Denklem 2'deki gibi olmaktadır.

$G S R=\operatorname{randn} \times \frac{2 \Delta x \times x_{n}}{\left(x_{\text {worst }}-x_{\text {best }}+\varepsilon\right)}$

Yerel kaçış operatörü, karmaşık problemlere karşı GBO algoritmasının verimliliğini arttırmak için kullanılmıştır.

\section{Deneysel Sonuçlar ve Tartışma}

GBO algoritmasına ait değerlerden elde edilen sonuçlar tablolar halinde verilmiştir. Aynı zamanda bunlara ait yakınsama grafikleri de şekiller bölümünde yer almaktadır. Fonksiyonlar ise tek modlu ve çok modlu fonksiyonlar olmak üzere ayrı ayrı tablolarda verilmiștir. Algoritmanın yineleme sayısı 1.000, boyut sayısı ise 25 olarak alınmıştır. Ayrıca algoritmanın parametre optimizasyonunun vektör sayıları üzerindeki etkisi de incelenmiştir. Bu sebep ile vektör sayıları sırası ile 10, 25 ve 50 alınmıştır.

Tablo 3. Tek modlu fonksiyonlar (vektör sayısı:10)

\begin{tabular}{|c|c|c|c|c|c|c|}
\hline & & 0.1 & 0.3 & 0.5 & 0.7 & 0.9 \\
\hline \multirow{3}{*}{$F 1$} & En iyi & $1.98 \mathrm{E}-70$ & $2.15 \mathrm{E}-147$ & $3.43 \mathrm{E}-202$ & $6.85 \mathrm{E}-264$ & $0.00 E+00$ \\
\hline & Ortalama & $2.40 \mathrm{E}-48$ & $2.60 \mathrm{E}-117$ & $8.14 \mathrm{E}-170$ & $3.76 \mathrm{E}-227$ & $4.16 \mathrm{E}-287$ \\
\hline & Std. & $1.28 \mathrm{E}-47$ & $1.40 \mathrm{E}-116$ & $0.00 \mathrm{E}+00$ & $0.00 \mathrm{E}+00$ & $0.00 \mathrm{E}+00$ \\
\hline \multirow{3}{*}{$F 2$} & En iyi & $2.34 \mathrm{E}-04$ & $1.85 E-04$ & $6.88 \mathrm{E}-05$ & $5.20 \mathrm{E}-05$ & $4.12 \mathrm{E}-05$ \\
\hline & Ortalama & $2.83 \mathrm{E}-03$ & $1.60 \mathrm{E}-03$ & $1.13 \mathrm{E}-03$ & $8.53 \mathrm{E}-04$ & $8.44 \mathrm{E}-04$ \\
\hline & Std. & $2.25 \mathrm{E}-03$ & $1.60 \mathrm{E}-03$ & $9.91 \mathrm{E}-04$ & $8.24 \mathrm{E}-04$ & $7.60 \mathrm{E}-04$ \\
\hline \multirow{3}{*}{$F 3$} & En iyi & $5.05 \mathrm{E}-149$ & $1.30 \mathrm{E}-259$ & $0.00 \mathrm{E}+00$ & $0.00 \mathrm{E}+00$ & $0.00 E+00$ \\
\hline & Ortalama & $1.69 \mathrm{E}-91$ & $2.81 \mathrm{E}-204$ & $1.31 \mathrm{E}-290$ & $0.00 \mathrm{E}+00$ & $0.00 \mathrm{E}+00$ \\
\hline & Std. & $8.67 \mathrm{E}-91$ & $0.00 \mathrm{E}+00$ & $0.00 \mathrm{E}+00$ & $0.00 \mathrm{E}+00$ & $0.00 \mathrm{E}+00$ \\
\hline \multirow{3}{*}{ F4 } & En iyi & $4.08 \mathrm{E}-41$ & $1.65 \mathrm{E}-77$ & $3.91 \mathrm{E}-102$ & $3.48 \mathrm{E}-129$ & $4.67 \mathrm{E}-161$ \\
\hline & Ortalama & $1.54 \mathrm{E}-22$ & $2.41 \mathrm{E}-62$ & $1.21 \mathrm{E}-88$ & $8.43 \mathrm{E}-113$ & $1.69 \mathrm{E}-144$ \\
\hline & Std. & $8.04 \mathrm{E}-22$ & $1.30 \mathrm{E}-61$ & 5.49E-88 & $4.54 \mathrm{E}-112$ & $6.89 \mathrm{E}-144$ \\
\hline \multirow{3}{*}{ F5 } & En iyi & $3.19 \mathrm{E}-32$ & $3.16 \mathrm{E}-66$ & $1.87 \mathrm{E}-92$ & $9.66 \mathrm{E}-129$ & $5.38 \mathrm{E}-164$ \\
\hline & Ortalama & $1.76 \mathrm{E}-21$ & $3.81 \mathrm{E}-52$ & $2.75 \mathrm{E}-81$ & $2.11 \mathrm{E}-113$ & $1.18 \mathrm{E}-143$ \\
\hline & Std. & $5.61 \mathrm{E}-21$ & $2.05 \mathrm{E}-51$ & $1.39 \mathrm{E}-80$ & $6.52 \mathrm{E}-113$ & $4.22 \mathrm{E}-143$ \\
\hline \multirow{3}{*}{ F6 } & En iyi & 9.80E-08 & 8.24E-09 & $3.11 \mathrm{E}-08$ & $1.33 \mathrm{E}-06$ & $1.33 \mathrm{E}-05$ \\
\hline & Ortalama & $9.69 \mathrm{E}-06$ & $7.81 \mathrm{E}-06$ & $2.58 \mathrm{E}-06$ & $1.29 \mathrm{E}-05$ & $2.50 \mathrm{E}-04$ \\
\hline & Std. & $2.00 \mathrm{E}-05$ & $2.73 \mathrm{E}-05$ & $4.17 \mathrm{E}-06$ & $1.61 \mathrm{E}-05$ & $2.46 \mathrm{E}-04$ \\
\hline
\end{tabular}


Avrupa Bilim ve Teknoloji Dergisi

\begin{tabular}{l|l|l|l|l|l|l}
\hline \multirow{4}{*}{$F 7$} & En iyi & $\mathbf{- 9 . 5 0 E}+\mathbf{0 1}$ & $-9.50 \mathrm{E}+01$ & $-9.50 \mathrm{E}+01$ & $-9.50 \mathrm{E}+01$ & $-9.50 \mathrm{E}+01$ \\
\cline { 2 - 7 } & Ortalama & $-9.50 \mathrm{E}+01$ & $-9.46 \mathrm{E}+01$ & $-9.43 \mathrm{E}+01$ & $-9.39 \mathrm{E}+01$ & $-9.28 \mathrm{E}+01$ \\
\cline { 2 - 7 } & Std. & $0.00 \mathrm{E}+00$ & $1.97 \mathrm{E}+00$ & $2.74 \mathrm{E}+00$ & $3.20 \mathrm{E}+00$ & $5.18 \mathrm{E}+00$ \\
\hline \multirow{4}{*}{$F 8$} & En iyi & $6.37 \mathrm{E}-55$ & $2.00 \mathrm{E}-121$ & $2.89 \mathrm{E}-178$ & $6.90 \mathrm{E}-251$ & $\mathbf{0 . 0 0 E}+\mathbf{0 0}$ \\
\cline { 2 - 7 } & Ortalama & $5.43 \mathrm{E}-33$ & $4.93 \mathrm{E}-93$ & $1.55 \mathrm{E}-153$ & $2.43 \mathrm{E}-208$ & $2.03 \mathrm{E}-283$ \\
\cline { 2 - 7 } & Std. & $2.87 \mathrm{E}-32$ & $2.66 \mathrm{E}-92$ & $6.26 \mathrm{E}-153$ & $0.00 \mathrm{E}+00$ & $0.00 \mathrm{E}+00$ \\
\hline \multirow{4}{*}{$F 9$} & En iyi & $2.22 \mathrm{E}-36$ & $1.83 \mathrm{E}-74$ & $1.34 \mathrm{E}-103$ & $4.04 \mathrm{E}-129$ & $\mathbf{2 . 3 1 E}-\mathbf{1 6 2}$ \\
\cline { 2 - 7 } & Ortalama & $9.78 \mathrm{E}-22$ & $1.10 \mathrm{E}-62$ & $2.72 \mathrm{E}-87$ & $6.30 \mathrm{E}-117$ & $1.37 \mathrm{E}-145$ \\
\cline { 2 - 7 } & Std. & $4.15 \mathrm{E}-21$ & $4.32 \mathrm{E}-62$ & $1.46 \mathrm{E}-86$ & $3.19 \mathrm{E}-116$ & $6.42 \mathrm{E}-145$ \\
\hline \multirow{4}{*}{$F 10$} & En iyi & $2.37 \mathrm{E}-315$ & $0.00 \mathrm{E}+00$ & $0.00 \mathrm{E}+00$ & $0.00 \mathrm{E}+00$ & $\mathbf{0 . 0 0 E}+\mathbf{0 0}$ \\
\cline { 2 - 7 } & Ortalama & $5.29 \mathrm{E}-193$ & $0.00 \mathrm{E}+00$ & $0.00 \mathrm{E}+00$ & $0.00 \mathrm{E}+00$ & $0.00 \mathrm{E}+00$ \\
\cline { 2 - 7 } & Std. & $0.00 \mathrm{E}+00$ & $0.00 \mathrm{E}+00$ & $0.00 \mathrm{E}+00$ & $0.00 \mathrm{E}+00$ & $0.00 \mathrm{E}+00$ \\
\hline \multirow{4}{*}{$F 12$} & En iyi & $1.46 \mathrm{E}+01$ & $\mathbf{1 . 3 8 E}+\mathbf{0 1}$ & $1.39 \mathrm{E}+01$ & $1.41 \mathrm{E}+01$ & $1.43 \mathrm{E}+01$ \\
\cline { 2 - 7 } & Ortalama & $1.59 \mathrm{E}+01$ & $1.57 \mathrm{E}+01$ & $1.59 \mathrm{E}+01$ & $1.61 \mathrm{E}+01$ & $1.62 \mathrm{E}+01$ \\
\cline { 2 - 7 } & Std. & $1.17 \mathrm{E}+00$ & $1.23 \mathrm{E}+00$ & $1.34 \mathrm{E}+00$ & $1.11 \mathrm{E}+00$ & $1.10 \mathrm{E}+00$ \\
\cline { 2 - 7 } & En iyi & $6.37 \mathrm{E}-76$ & $1.67 \mathrm{E}-150$ & $1.18 \mathrm{E}-205$ & $2.78 \mathrm{E}-262$ & $\mathbf{0 . 0 0 E}+\mathbf{0 0}$ \\
\cline { 2 - 7 } & Ortalama & $1.23 \mathrm{E}-45$ & $2.93 \mathrm{E}-123$ & $2.19 \mathrm{E}-172$ & $6.01 \mathrm{E}-230$ & $2.99 \mathrm{E}-293$ \\
\cline { 2 - 7 } & Std. & $6.62 \mathrm{E}-45$ & $1.58 \mathrm{E}-122$ & $0.00 \mathrm{E}+00$ & $0.00 \mathrm{E}+00$ & $0.00 \mathrm{E}+00$ \\
\hline
\end{tabular}
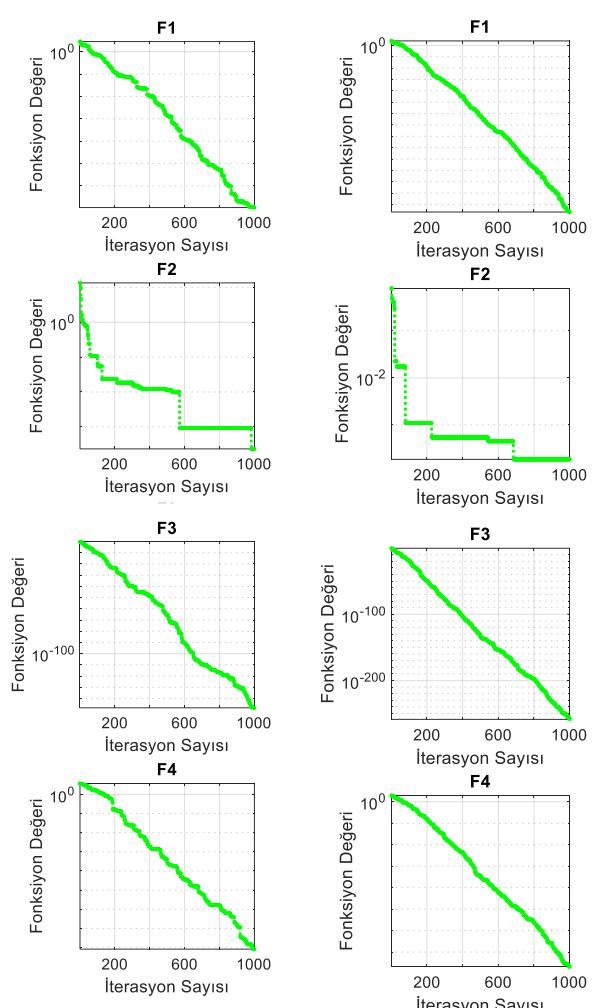

F5

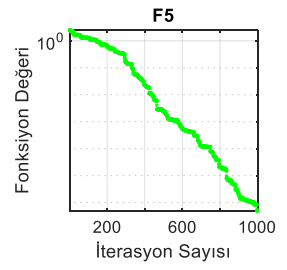

F
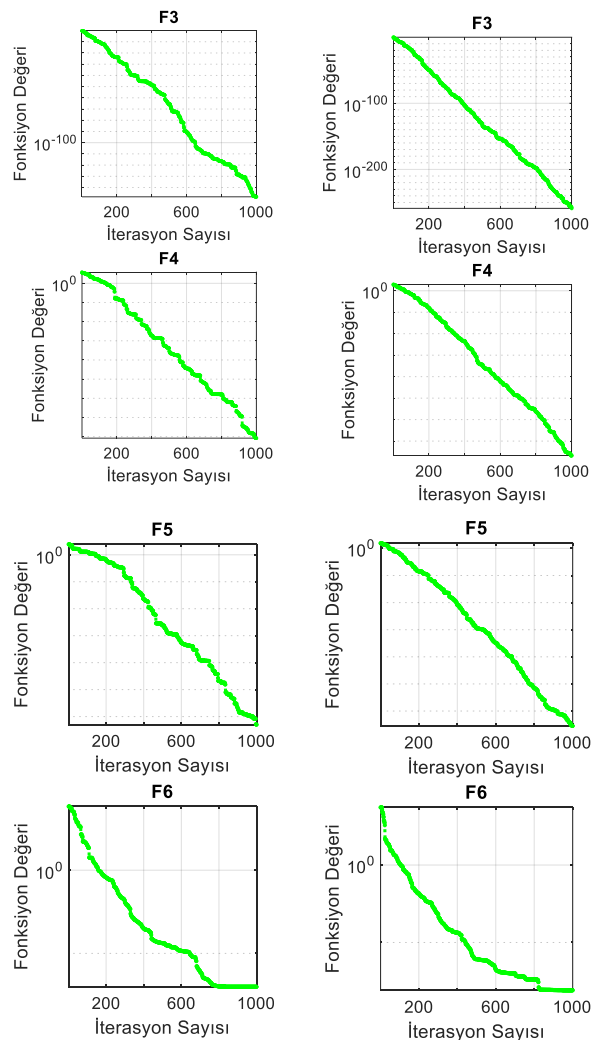

F5

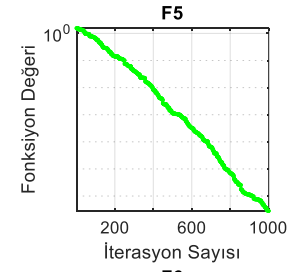

F6

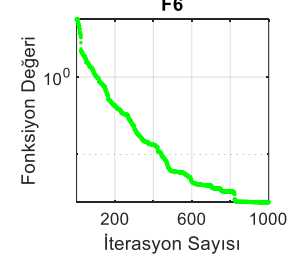

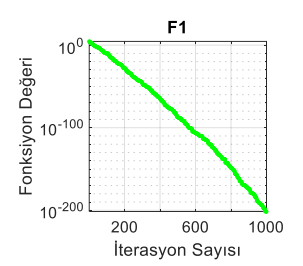
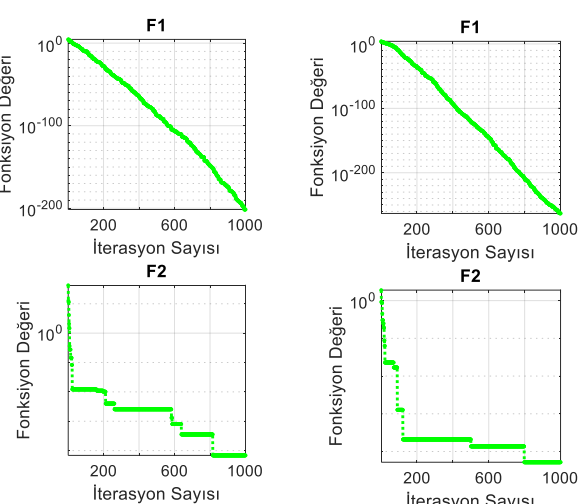

F3
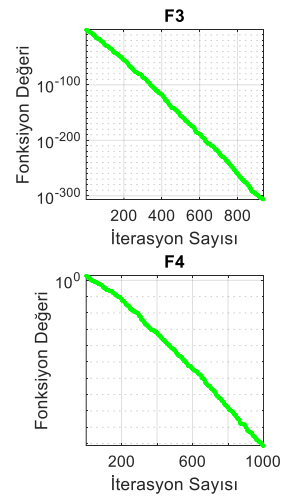

F5

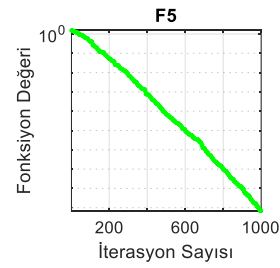

F6

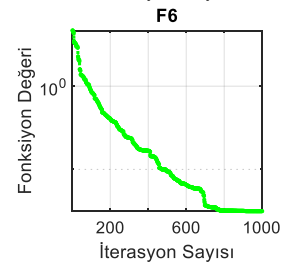

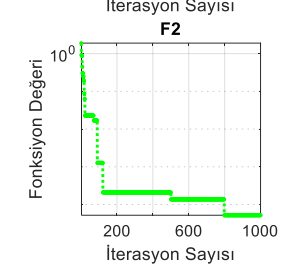

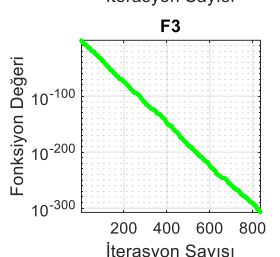

Iterasyon Sayıs!

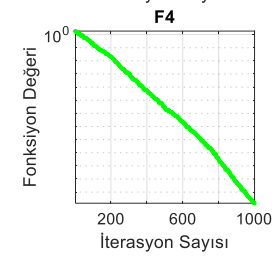

F5
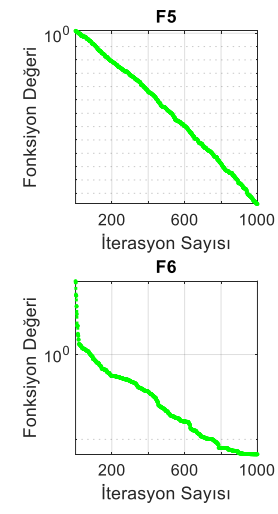

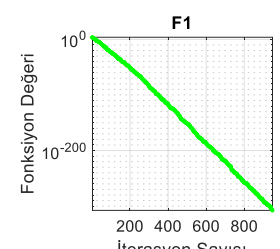

Iterasyon Sayısı

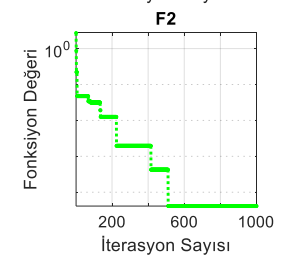

F3
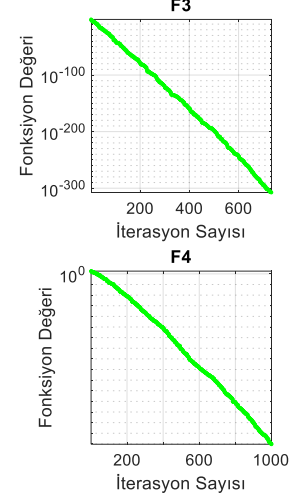

F5

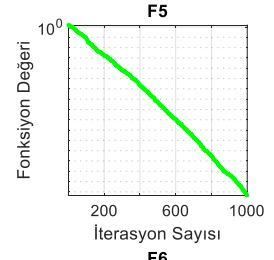

F6

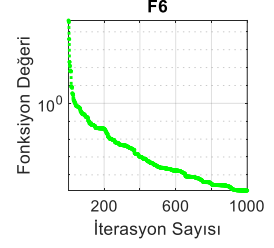


European Journal of Science and Technology
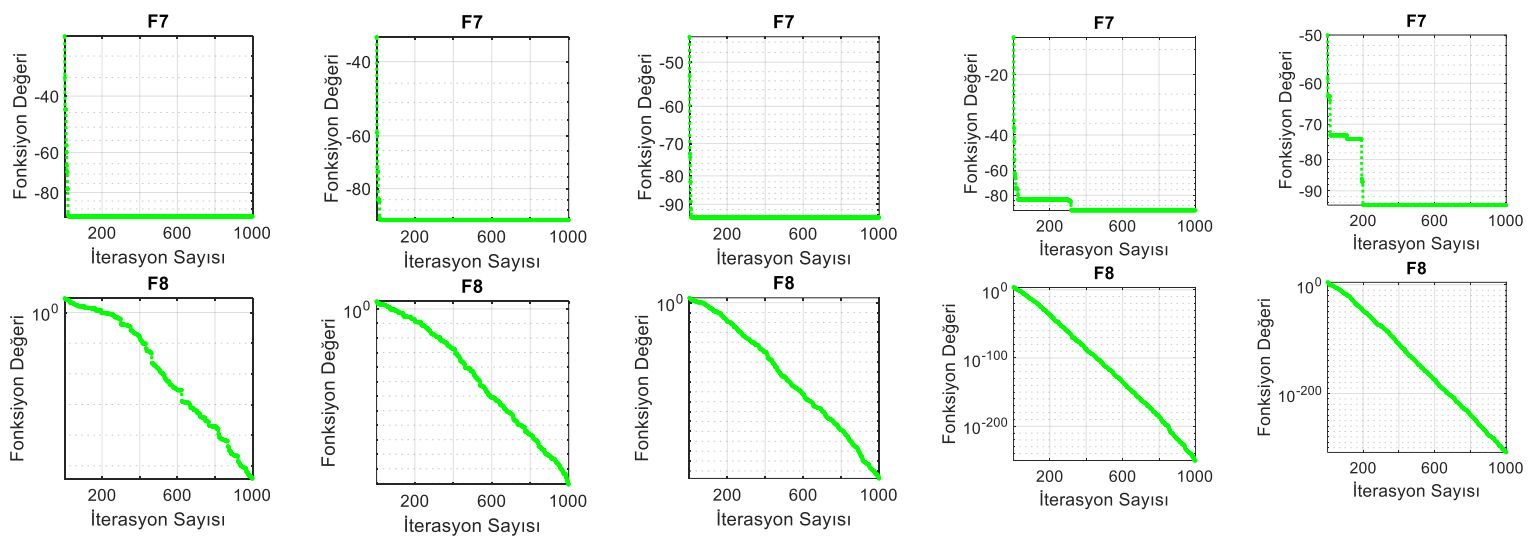

F9
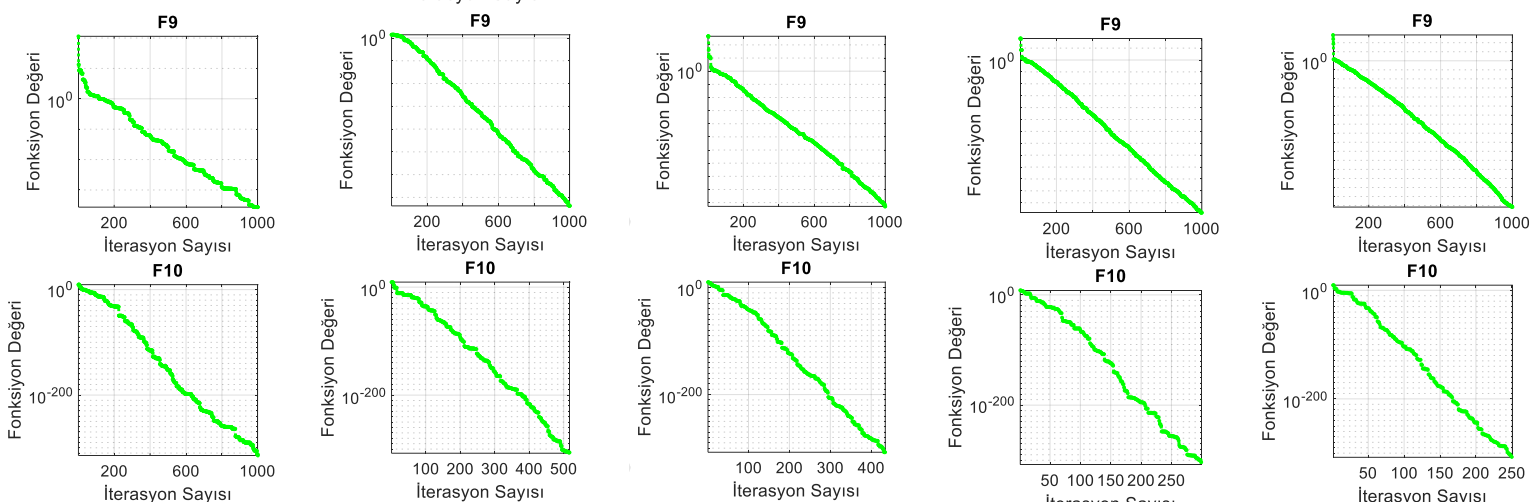

F10
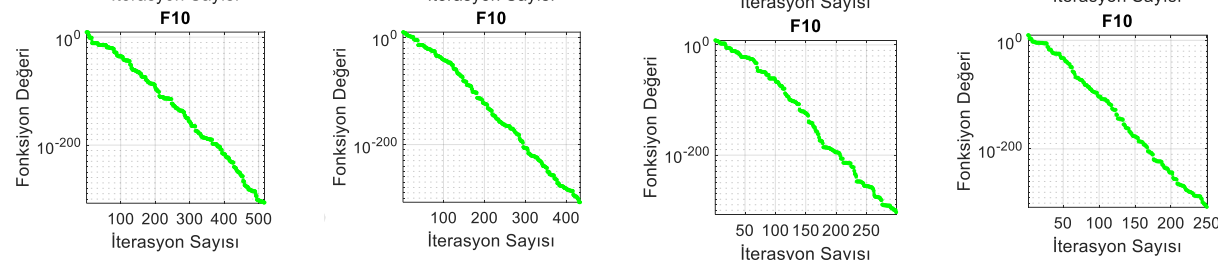

F11

F11
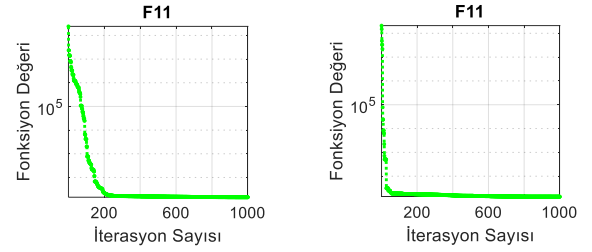

F11

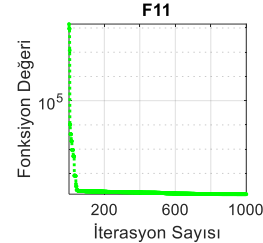

F11
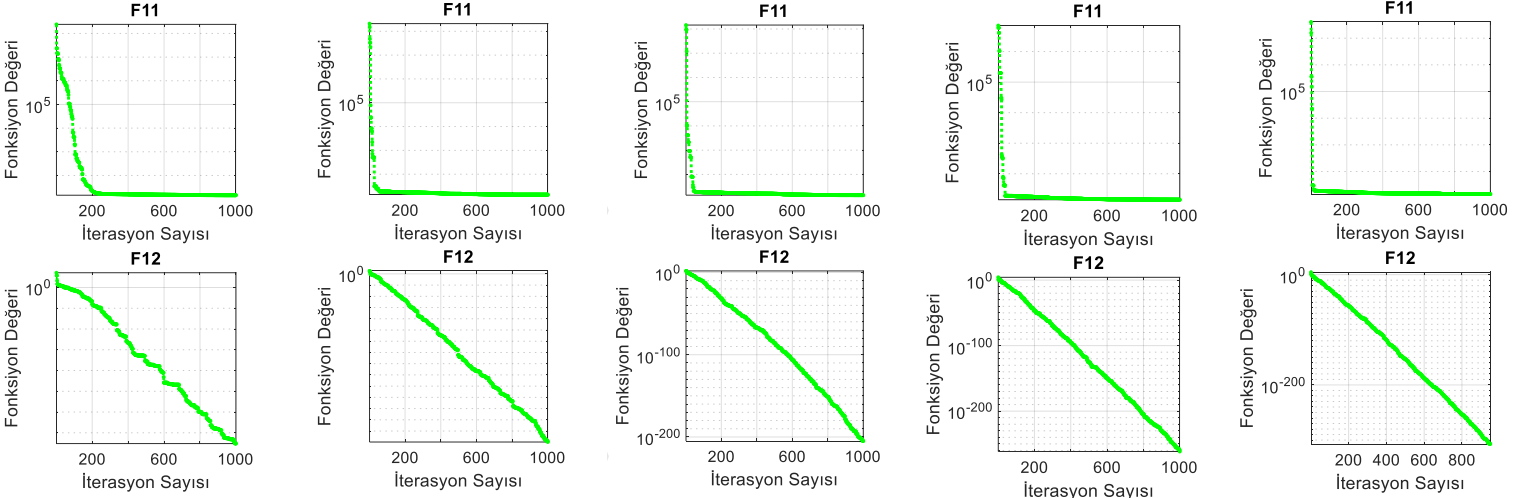

Şekil 1. Tek modlu fonksiyonlar (vektör saylsı:10)

Tablo 4. Çok modlu fonksiyonlar (vektör sayısı:10)

\begin{tabular}{c|l|l|l|l|l|c}
\hline & & \multicolumn{1}{|c|}{$\mathbf{0 . 1}$} & \multicolumn{1}{|c|}{} & \multicolumn{1}{c|}{$\mathbf{0 . 5}$} & $\mathbf{0 . 7}$ & $\mathbf{0 . 9}$ \\
\hline \multirow{5}{*}{$F 1$} & En iyi & $6.82 \mathrm{E}+01$ & $3.55 \mathrm{E}+01$ & $\mathbf{1 . 1 8 E}+\mathbf{0 1}$ & $7.02 \mathrm{E}+01$ & $5.53 \mathrm{E}+01$ \\
\cline { 2 - 7 } & Ortalama & $1.25 \mathrm{E}+02$ & $1.20 \mathrm{E}+02$ & $1.30 \mathrm{E}+02$ & $1.42 \mathrm{E}+02$ & $1.47 \mathrm{E}+02$ \\
\cline { 2 - 7 } & Std. & $2.50 \mathrm{E}+01$ & $3.84 \mathrm{E}+01$ & $3.76 \mathrm{E}+01$ & $3.13 \mathrm{E}+01$ & $3.93 \mathrm{E}+01$ \\
\hline \multirow{5}{*}{$F 2$} & En iyi & $0.00 \mathrm{E}+00$ & $0.00 \mathrm{E}+00$ & $0.00 \mathrm{E}+00$ & $0.00 \mathrm{E}+00$ & $0.00 \mathrm{E}+00$ \\
\cline { 2 - 7 } & Ortalama & $3.32 \mathrm{E}-02$ & $0.00 \mathrm{E}+00$ & $0.00 \mathrm{E}+00$ & $0.00 \mathrm{E}+00$ & $0.00 \mathrm{E}+00$ \\
\cline { 2 - 7 } & Std. & $1.79 \mathrm{E}-01$ & $0.00 \mathrm{E}+00$ & $0.00 \mathrm{E}+00$ & $0.00 \mathrm{E}+00$ & $0.00 \mathrm{E}+00$ \\
\hline \multirow{5}{*}{$F 3$} & En iyi & $9.00 \mathrm{E}-01$ & $9.00 \mathrm{E}-01$ & $9.00 \mathrm{E}-01$ & $9.00 \mathrm{E}-01$ & $9.00 \mathrm{E}-01$ \\
\cline { 2 - 7 } & Ortalama & $9.84 \mathrm{E}-01$ & $9.20 \mathrm{E}-01$ & $9.03 \mathrm{E}-01$ & $9.00 \mathrm{E}-01$ & $9.00 \mathrm{E}-01$ \\
\cline { 2 - 7 } & Std. & $6.93 \mathrm{E}-02$ & $4.01 \mathrm{E}-02$ & $1.81 \mathrm{E}-02$ & $4.44 \mathrm{E}-16$ & $4.44 \mathrm{E}-16$ \\
\hline \multirow{5}{*}{$F 4$} & En iyi & $9.67 \mathrm{E}-05$ & $\mathbf{1 . 3 3 E}-\mathbf{0 5}$ & $2.11 \mathrm{E}-04$ & $1.22 \mathrm{E}-03$ & $5.87 \mathrm{E}-02$ \\
\cline { 2 - 7 } & Ortalama & $1.60 \mathrm{E}-02$ & $7.23 \mathrm{E}-02$ & $2.98 \mathrm{E}-02$ & $9.57 \mathrm{E}-02$ & $4.98 \mathrm{E}+00$ \\
\cline { 2 - 7 } & Std. & $3.15 \mathrm{E}-02$ & $3.52 \mathrm{E}-01$ & $7.80 \mathrm{E}-02$ & $2.34 \mathrm{E}-01$ & $9.83 \mathrm{E}+00$ \\
\hline \multirow{3}{*}{$F 5$} & En iyi & $3.86 \mathrm{E}-39$ & $1.44 \mathrm{E}-73$ & $1.56 \mathrm{E}-103$ & $1.89 \mathrm{E}-135$ & $\mathbf{1 . 9 2 E}-161$ \\
\cline { 2 - 7 } & Ortalama & $9.19 \mathrm{E}-05$ & $1.30 \mathrm{E}-05$ & $4.01 \mathrm{E}-91$ & $1.31 \mathrm{E}-116$ & $9.37 \mathrm{E}-148$ \\
\cline { 2 - 7 } & Std. & $1.90 \mathrm{E}-04$ & $7.01 \mathrm{E}-05$ & $2.15 \mathrm{E}-90$ & $7.07 \mathrm{E}-116$ & $4.99 \mathrm{E}-147$ \\
\hline$F 6$ & En iyi & $3.88 \mathrm{E}-39$ & $5.79 \mathrm{E}-62$ & $1.79 \mathrm{E}-85$ & $1.12 \mathrm{E}-99$ & $\mathbf{1 . 0 6 E}-108$ \\
\hline
\end{tabular}


Avrupa Bilim ve Teknoloji Dergisi

\begin{tabular}{|c|c|c|c|c|c|c|}
\hline & Ortalama & $4.09 \mathrm{E}-13$ & $1.09 \mathrm{E}-28$ & $1.55 \mathrm{E}-22$ & $8.49 \mathrm{E}-24$ & $1.16 \mathrm{E}-28$ \\
\hline & Std. & $1.53 \mathrm{E}-12$ & $5.88 \mathrm{E}-28$ & $8.37 \mathrm{E}-22$ & $4.57 \mathrm{E}-23$ & $4.57 \mathrm{E}-28$ \\
\hline \multirow{3}{*}{$F 7$} & En iyi & $0.00 \mathrm{E}+00$ & $0.00 \mathrm{E}+00$ & $0.00 \mathrm{E}+00$ & $0.00 \mathrm{E}+00$ & $0.00 \mathrm{E}+00$ \\
\hline & Ortalama & $0.00 \mathrm{E}+00$ & $0.00 \mathrm{E}+00$ & $0.00 \mathrm{E}+00$ & $0.00 \mathrm{E}+00$ & $0.00 \mathrm{E}+00$ \\
\hline & Std. & $0.00 \mathrm{E}+00$ & $0.00 \mathrm{E}+00$ & $0.00 \mathrm{E}+00$ & $0.00 \mathrm{E}+00$ & $0.00 \mathrm{E}+00$ \\
\hline \multirow{3}{*}{$F 8$} & En iyi & $1.94 \mathrm{E}+00$ & $3.24 \mathrm{E}+00$ & $4.59 \mathrm{E}+00$ & $9.52 \mathrm{E}+00$ & $1.10 \mathrm{E}+01$ \\
\hline & Ortalama & $1.11 \mathrm{E}+01$ & $1.24 \mathrm{E}+01$ & $1.70 \mathrm{E}+01$ & $2.17 \mathrm{E}+01$ & $2.31 \mathrm{E}+01$ \\
\hline & Std. & $5.44 \mathrm{E}+00$ & $5.88 \mathrm{E}+00$ & $7.92 \mathrm{E}+00$ & $9.06 \mathrm{E}+00$ & $8.13 \mathrm{E}+00$ \\
\hline \multirow{3}{*}{ F9 } & En iyi & $5.02 \mathrm{E}-24$ & $2.10 \mathrm{E}-66$ & $7.84 \mathrm{E}-93$ & $5.28 \mathrm{E}-125$ & $3.30 \mathrm{E}-160$ \\
\hline & Ortalama & $3.33 \mathrm{E}-02$ & $3.33 \mathrm{E}-03$ & $1.45 \mathrm{E}-62$ & $2.10 \mathrm{E}-83$ & $1.09 \mathrm{E}-127$ \\
\hline & Std. & $4.70 \mathrm{E}-02$ & 1.79E-02 & $7.81 \mathrm{E}-62$ & $8.74 \mathrm{E}-83$ & $5.86 \mathrm{E}-127$ \\
\hline \multirow{3}{*}{ F10 } & En iyi & $-7.83 \mathrm{E}+02$ & $-7.55 \mathrm{E}+02$ & $-7.55 \mathrm{E}+02$ & $-7.41 \mathrm{E}+02$ & $-7.41 \mathrm{E}+02$ \\
\hline & Ortalama & $-7.05 \mathrm{E}+02$ & $-6.97 \mathrm{E}+02$ & $-6.95 \mathrm{E}+02$ & $-6.83 \mathrm{E}+02$ & $-6.81 \mathrm{E}+02$ \\
\hline & Std. & $3.51 \mathrm{E}+01$ & $3.37 \mathrm{E}+01$ & $2.72 \mathrm{E}+01$ & $2.62 \mathrm{E}+01$ & $2.36 \mathrm{E}+01$ \\
\hline \multirow{3}{*}{ F11 } & En iyi & $0.00 \mathrm{E}+00$ & $0.00 \mathrm{E}+00$ & $0.00 \mathrm{E}+00$ & $0.00 \mathrm{E}+00$ & $0.00 \mathrm{E}+00$ \\
\hline & Ortalama & $0.00 \mathrm{E}+00$ & $0.00 \mathrm{E}+00$ & $0.00 \mathrm{E}+00$ & $0.00 \mathrm{E}+00$ & $0.00 \mathrm{E}+00$ \\
\hline & Std. & $0.00 \mathrm{E}+00$ & $0.00 \mathrm{E}+00$ & $0.00 \mathrm{E}+00$ & $0.00 \mathrm{E}+00$ & $0.00 \mathrm{E}+00$ \\
\hline \multirow{3}{*}{$F 12$} & En iyi & $-1.00 \mathrm{E}+00$ & $-1.00 \mathrm{E}+00$ & $-1.00 \mathrm{E}+00$ & $-1.00 \mathrm{E}+00$ & $-1.00 \mathrm{E}+00$ \\
\hline & Ortalama & $-9.19 \mathrm{E}-01$ & $-1.00 \mathrm{E}+00$ & $-1.00 \mathrm{E}+00$ & $-1.00 \mathrm{E}+00$ & $-1.00 \mathrm{E}+00$ \\
\hline & Std. & $2.56 \mathrm{E}-01$ & $0.00 \mathrm{E}+00$ & $0.00 \mathrm{E}+00$ & $0.00 \mathrm{E}+00$ & $0.00 \mathrm{E}+00$ \\
\hline
\end{tabular}

F1

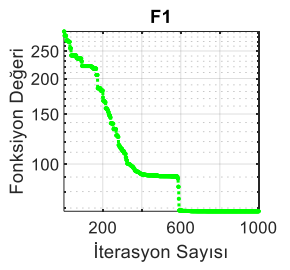

F2

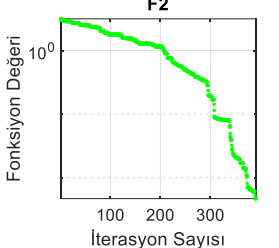

F3
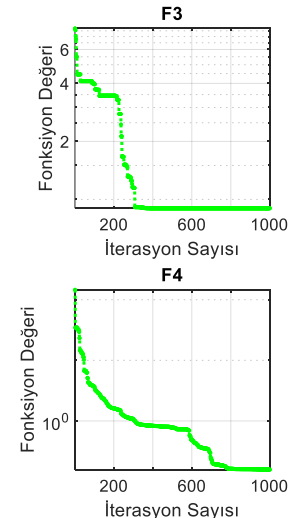

F5

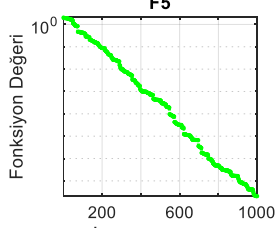

Iterasyon Sayısı

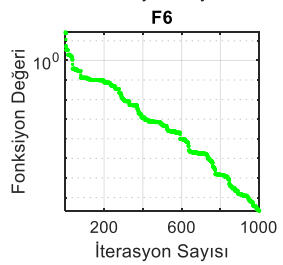

F1
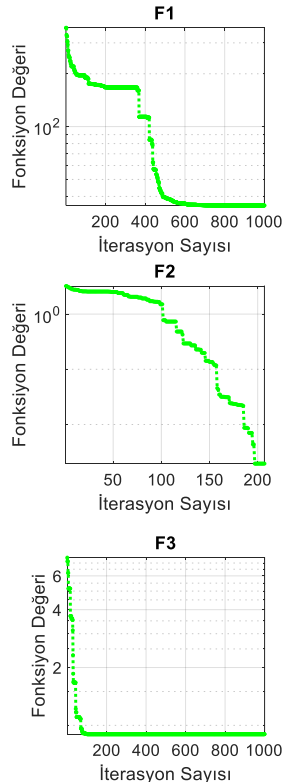

F4
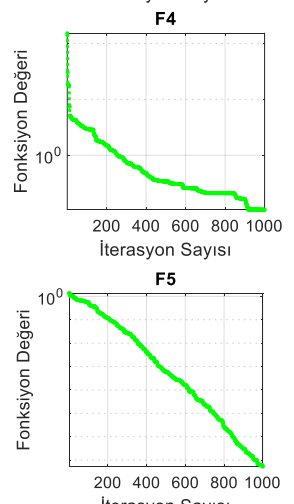

Iterasyon Sayısı

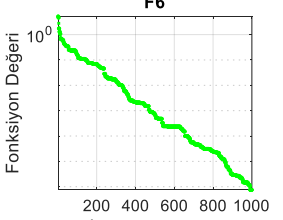

Iterasyon Sayısı
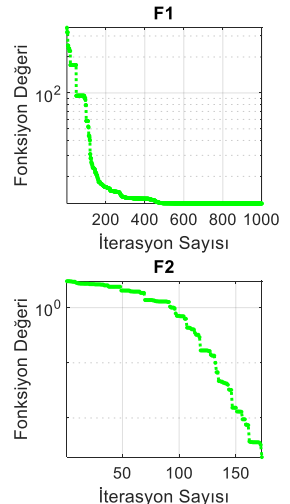

F3

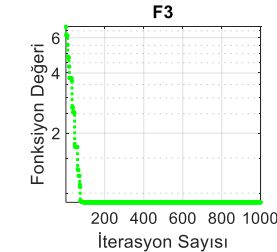

F4
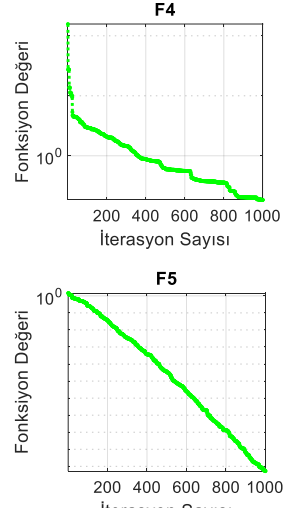

Iterasyon Sayıs!

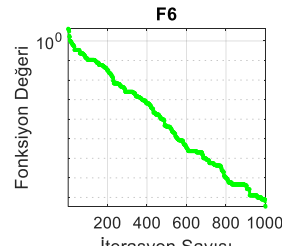

Iterasyon Sayıs!
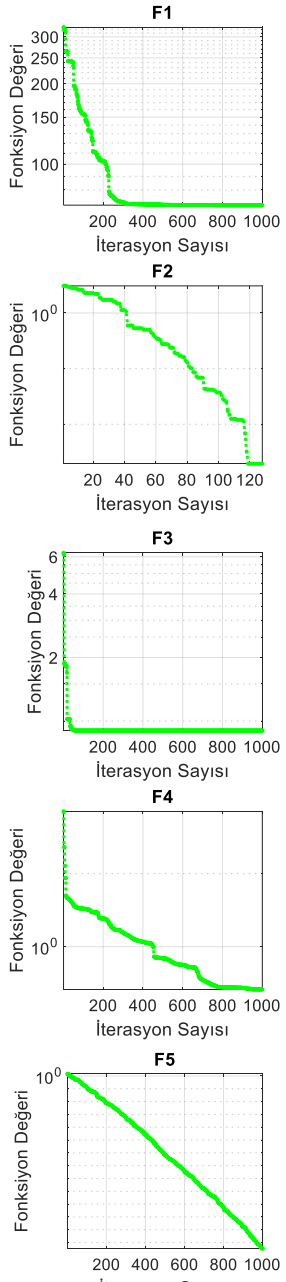

Iterasyon Sayısı

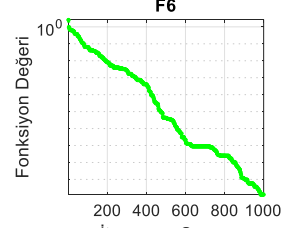

200400600800
Iterasyon Sayıs

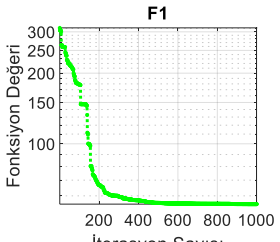

Iterasyon Sayısı

F2
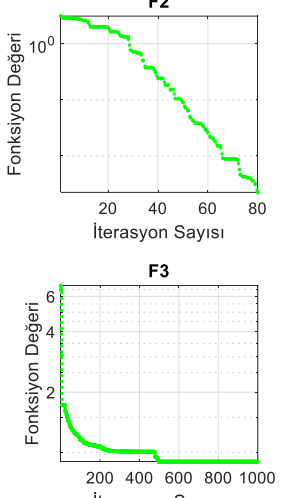

Iterasyon Sayıs।
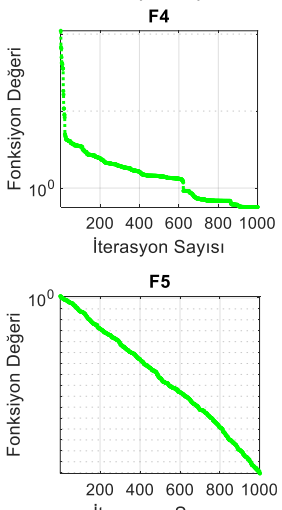

F6

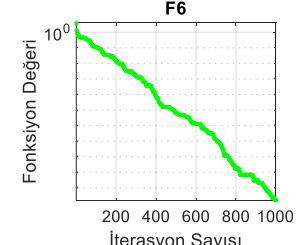

$200 \quad 400600800$
Iterasyon Sayıs! 
European Journal of Science and Technology
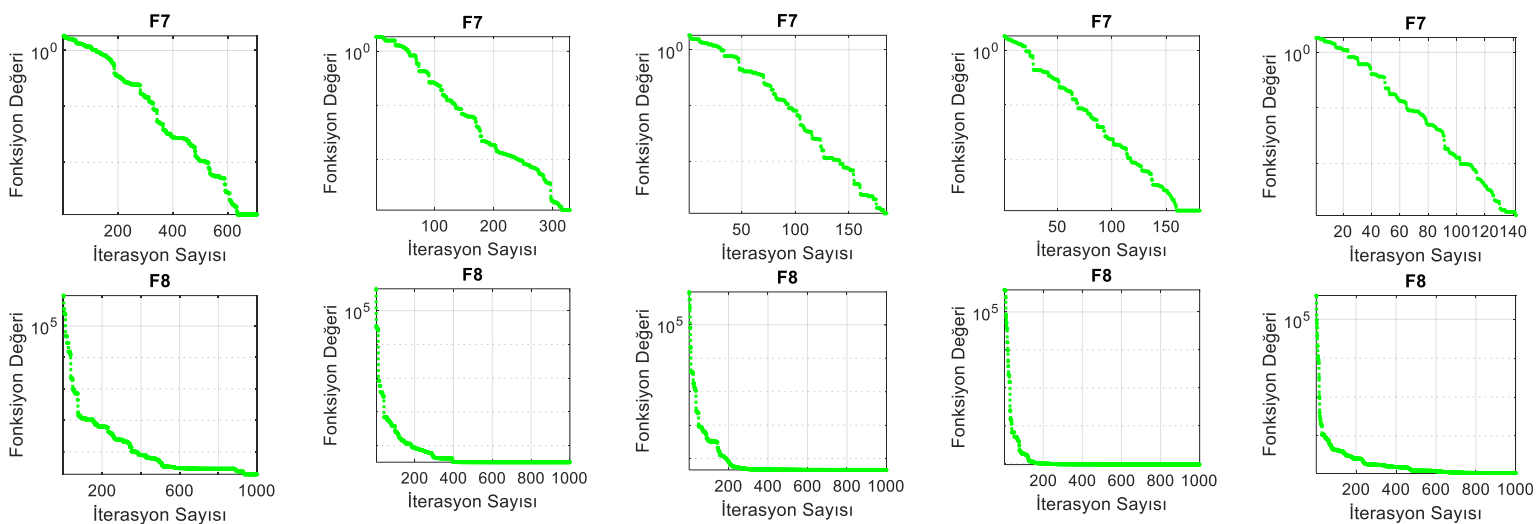

F9

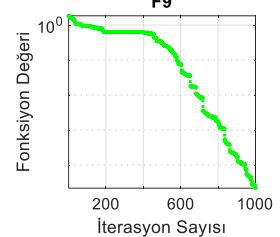

F9
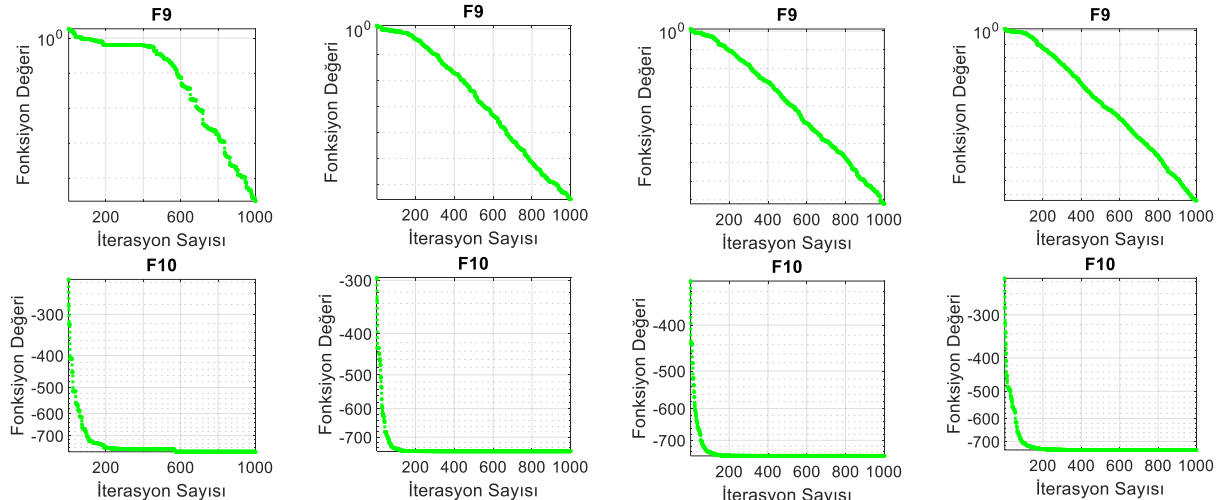

F10

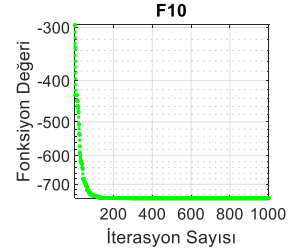

F10

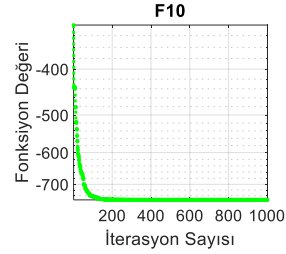

F10
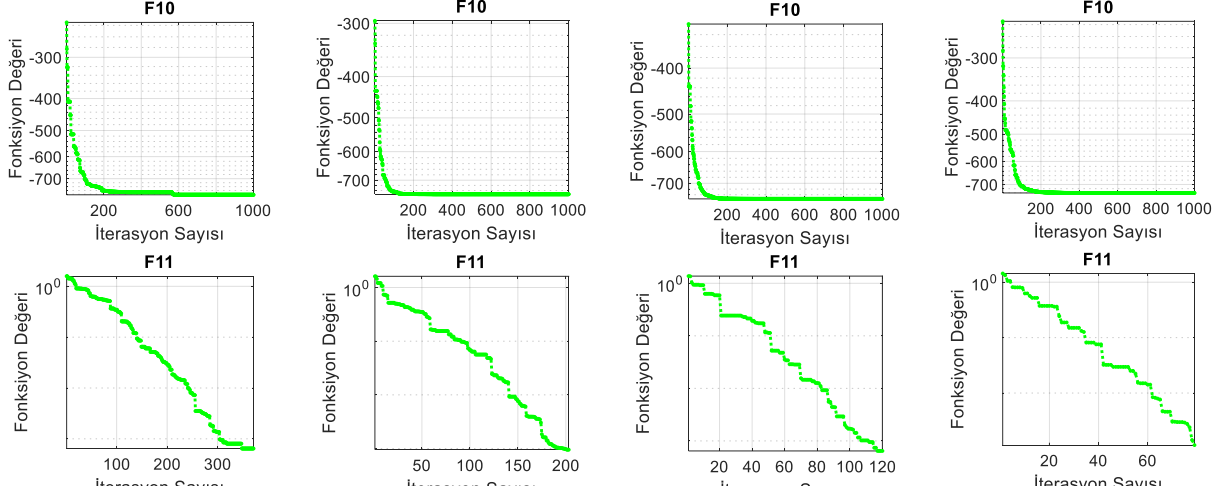

F11

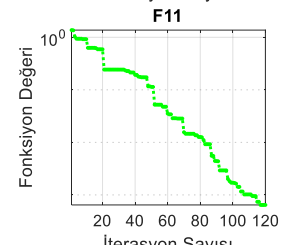

F11
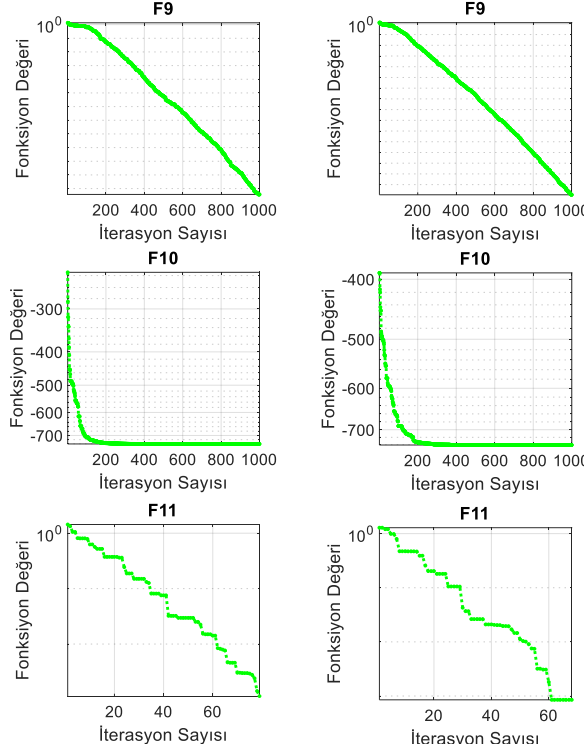

Iterasyon Sayısı

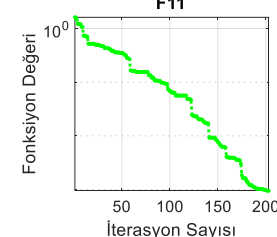

F12
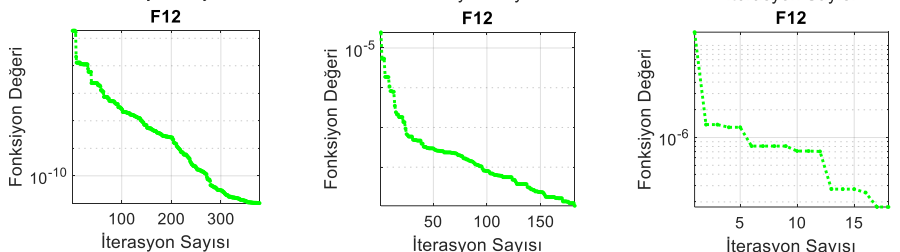

F12

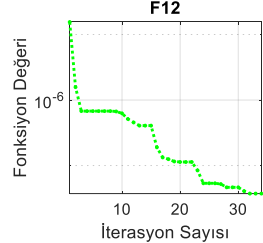

Şekil 2. Çok modlu fonksiyonlar (vektör saylsı:10)
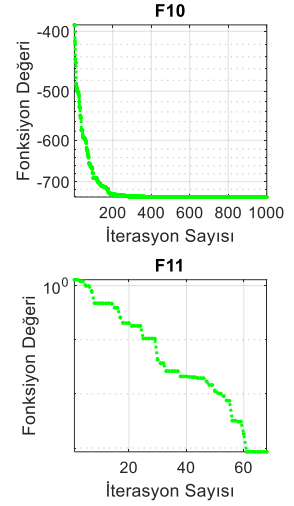

F12

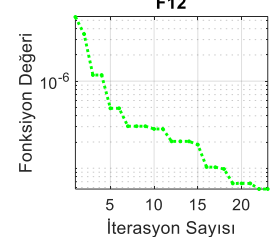

Tablo 5. Tek modlu fonksiyonlar (vektör saylsı:25)

\begin{tabular}{|c|c|c|c|c|c|c|}
\hline & & $\begin{array}{l}0.1 \\
\end{array}$ & 0.3 & 0.5 & 0.7 & 0.9 \\
\hline \multirow{3}{*}{$F 1$} & En iyi & $1.50 \mathrm{E}-120$ & $1.77 \mathrm{E}-199$ & $7.39 \mathrm{E}-259$ & $2.00 \mathrm{E}-323$ & $0.00 \mathrm{E}+00$ \\
\hline & Ortalama & $1.16 \mathrm{E}-92$ & $1.13 \mathrm{E}-176$ & $1.76 \mathrm{E}-239$ & $1.97 \mathrm{E}-297$ & $0.00 \mathrm{E}+00$ \\
\hline & Std. & $6.22 \mathrm{E}-92$ & $0.00 \mathrm{E}+00$ & $0.00 \mathrm{E}+00$ & $0.00 \mathrm{E}+00$ & $0.00 \mathrm{E}+00$ \\
\hline \multirow{3}{*}{$F 2$} & En iyi & $6.68 \mathrm{E}-05$ & $2.50 \mathrm{E}-04$ & $3.11 \mathrm{E}-05$ & $1.07 \mathrm{E}-05$ & $7.50 \mathrm{E}-06$ \\
\hline & Ortalama & $1.23 \mathrm{E}-03$ & 7.10E-04 & $4.16 \mathrm{E}-04$ & $5.10 \mathrm{E}-04$ & $3.21 \mathrm{E}-04$ \\
\hline & Std. & $8.58 \mathrm{E}-04$ & $4.45 \mathrm{E}-04$ & $3.37 \mathrm{E}-04$ & $5.00 \mathrm{E}-04$ & $3.26 \mathrm{E}-04$ \\
\hline \multirow{3}{*}{$F 3$} & En iyi & $2.16 \mathrm{E}-225$ & $0.00 \mathrm{E}+00$ & $0.00 \mathrm{E}+00$ & $0.00 \mathrm{E}+00$ & $0.00 \mathrm{E}+00$ \\
\hline & Ortalama & $4.93 \mathrm{E}-189$ & $2.85 \mathrm{E}-314$ & $0.00 \mathrm{E}+00$ & $0.00 \mathrm{E}+00$ & $0.00 \mathrm{E}+00$ \\
\hline & Std. & $0.00 \mathrm{E}+00$ & $0.00 \mathrm{E}+00$ & $0.00 \mathrm{E}+00$ & $0.00 \mathrm{E}+00$ & $0.00 \mathrm{E}+00$ \\
\hline \multirow{3}{*}{$F 4$} & En iyi & $3.72 \mathrm{E}-60$ & $8.82 \mathrm{E}-100$ & $7.88 \mathrm{E}-128$ & $7.65 \mathrm{E}-160$ & $3.66 \mathrm{E}-193$ \\
\hline & Ortalama & $2.95 \mathrm{E}-53$ & $2.76 \mathrm{E}-92$ & $6.91 \mathrm{E}-119$ & $1.52 \mathrm{E}-150$ & $1.75 \mathrm{E}-181$ \\
\hline & Std. & $9.71 \mathrm{E}-53$ & $1.11 \mathrm{E}-91$ & $3.67 \mathrm{E}-118$ & $5.89 \mathrm{E}-150$ & $0.00 \mathrm{E}+00$ \\
\hline \multirow{3}{*}{$F 5$} & En iyi & $1.78 \mathrm{E}-47$ & $4.82 \mathrm{E}-87$ & $1.08 \mathrm{E}-119$ & $2.76 \mathrm{E}-151$ & 3.43E-191 \\
\hline & Ortalama & $2.86 \mathrm{E}-39$ & $3.82 \mathrm{E}-77$ & $3.09 \mathrm{E}-112$ & $1.16 \mathrm{E}-140$ & $4.23 \mathrm{E}-181$ \\
\hline & Std. & $1.30 \mathrm{E}-38$ & $1.95 \mathrm{E}-76$ & $6.40 \mathrm{E}-112$ & $4.84 \mathrm{E}-140$ & $0.00 \mathrm{E}+00$ \\
\hline \multirow{2}{*}{ F6 } & En iyi & $3.62 \mathrm{E}-19$ & $2.29 \mathrm{E}-19$ & $6.67 \mathrm{E}-17$ & $4.89 \mathrm{E}-14$ & $2.41 \mathrm{E}-10$ \\
\hline & Ortalama & $5.20 \mathrm{E}-16$ & $2.13 \mathrm{E}-16$ & $1.43 \mathrm{E}-13$ & $3.50 \mathrm{E}-11$ & $4.59 \mathrm{E}-08$ \\
\hline
\end{tabular}


Avrupa Bilim ve Teknoloji Dergisi

\begin{tabular}{c|l|l|l|l|l|l}
\hline & Std. & $1.77 \mathrm{E}-15$ & $4.73 \mathrm{E}-16$ & $5.18 \mathrm{E}-13$ & $1.02 \mathrm{E}-10$ & $1.71 \mathrm{E}-07$ \\
\hline \multirow{5}{*}{$F 7$} & En iyi & $-9.50 \mathrm{E}+01$ & $-9.50 \mathrm{E}+01$ & $-9.50 \mathrm{E}+01$ & $-9.50 \mathrm{E}+01$ & $-9.50 \mathrm{E}+01$ \\
\cline { 2 - 7 } & Ortalama & $-9.50 \mathrm{E}+01$ & $-9.50 \mathrm{E}+01$ & $-9.50 \mathrm{E}+01$ & $-9.46 \mathrm{E}+01$ & $-9.50 \mathrm{E}+01$ \\
\cline { 2 - 7 } & Std. & $0.00 \mathrm{E}+00$ & $0.00 \mathrm{E}+00$ & $0.00 \mathrm{E}+00$ & $1.97 \mathrm{E}+00$ & $0.00 \mathrm{E}+00$ \\
\hline \multirow{5}{*}{$F 8$} & En iyi & $7.22 \mathrm{E}-78$ & $1.49 \mathrm{E}-150$ & $2.77 \mathrm{E}-222$ & $2.86 \mathrm{E}-299$ & $\mathbf{0 . 0 0 E}+\mathbf{0 0}$ \\
\cline { 2 - 7 } & Ortalama & $5.86 \mathrm{E}-62$ & $2.29 \mathrm{E}-132$ & $2.42 \mathrm{E}-195$ & $3.44 \mathrm{E}-277$ & $0.00 \mathrm{E}+00$ \\
\cline { 2 - 7 } & Std. & $2.20 \mathrm{E}-61$ & $9.46 \mathrm{E}-132$ & $0.00 \mathrm{E}+00$ & $0.00 \mathrm{E}+00$ & $0.00 \mathrm{E}+00$ \\
\hline \multirow{5}{*}{$F 9$} & En iyi & $9.93 \mathrm{E}-61$ & $1.18 \mathrm{E}-98$ & $2.85 \mathrm{E}-129$ & $2.08 \mathrm{E}-158$ & $\mathbf{3 . 1 4 E}-194$ \\
\cline { 2 - 7 } & Ortalama & $3.14 \mathrm{E}-47$ & $7.93 \mathrm{E}-91$ & $4.03 \mathrm{E}-117$ & $2.01 \mathrm{E}-150$ & $9.62 \mathrm{E}-183$ \\
\cline { 2 - 7 } & Std. & $1.69 \mathrm{E}-46$ & $2.75 \mathrm{E}-90$ & $2.17 \mathrm{E}-116$ & $7.71 \mathrm{E}-150$ & $0.00 \mathrm{E}+00$ \\
\hline \multirow{5}{*}{$F 10$} & En iyi & $0.00 \mathrm{E}+00$ & $0.00 \mathrm{E}+00$ & $0.00 \mathrm{E}+00$ & $0.00 \mathrm{E}+00$ & $0.00 \mathrm{E}+00$ \\
\cline { 2 - 7 } & Ortalama & $0.00 \mathrm{E}+00$ & $0.00 \mathrm{E}+00$ & $0.00 \mathrm{E}+00$ & $0.00 \mathrm{E}+00$ & $0.00 \mathrm{E}+00$ \\
\cline { 2 - 7 } & Std. & $0.00 \mathrm{E}+00$ & $0.00 \mathrm{E}+00$ & $0.00 \mathrm{E}+00$ & $0.00 \mathrm{E}+00$ & $0.00 \mathrm{E}+00$ \\
\hline \multirow{3}{*}{$F 12$} & En iyi & $1.02 \mathrm{E}+01$ & $8.81 \mathrm{E}+00$ & $\mathbf{7 . 2 5 E}+\mathbf{0 0}$ & $9.23 \mathrm{E}+00$ & $1.05 \mathrm{E}+01$ \\
\cline { 2 - 7 } & Ortalama & $1.25 \mathrm{E}+01$ & $1.15 \mathrm{E}+01$ & $1.08 \mathrm{E}+01$ & $1.11 \mathrm{E}+01$ & $1.27 \mathrm{E}+01$ \\
\cline { 2 - 7 } & Std. & $1.27 \mathrm{E}+00$ & $2.08 \mathrm{E}+00$ & $1.79 \mathrm{E}+00$ & $1.58 \mathrm{E}+00$ & $1.40 \mathrm{E}+00$ \\
\hline & En iyi & $1.87 \mathrm{E}-124$ & $5.48 \mathrm{E}-203$ & $8.14 \mathrm{E}-258$ & $2.32 \mathrm{E}-320$ & $\mathbf{0 . 0 0 E}+\mathbf{0 0}$ \\
\cline { 2 - 7 } & Ortalama & $3.66 \mathrm{E}-102$ & $1.33 \mathrm{E}-181$ & $2.08 \mathrm{E}-241$ & $3.04 \mathrm{E}-303$ & $0.00 \mathrm{E}+00$ \\
\cline { 2 - 7 } & Std. & $1.79 \mathrm{E}-101$ & $0.00 \mathrm{E}+00$ & $0.00 \mathrm{E}+00$ & $0.00 \mathrm{E}+00$ & $0.00 \mathrm{E}+00$ \\
\hline
\end{tabular}
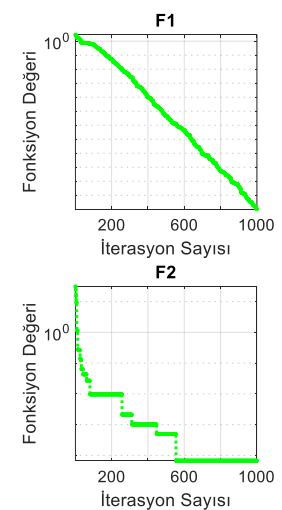

F3

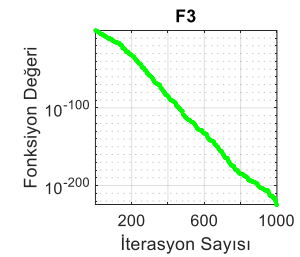

F4
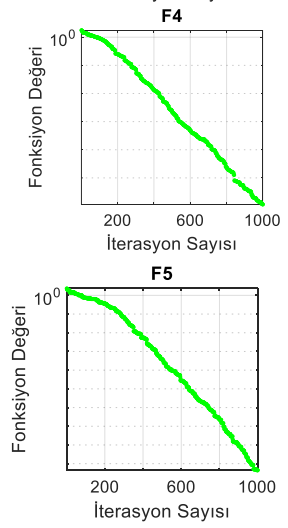

F6

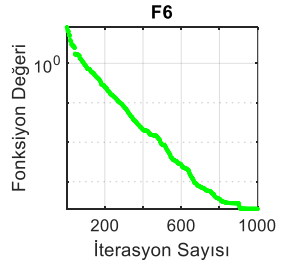

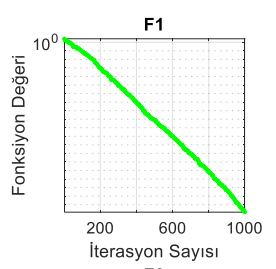

F2

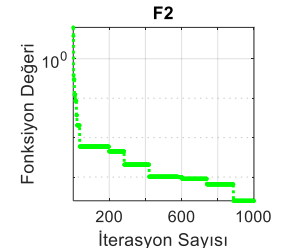

F3
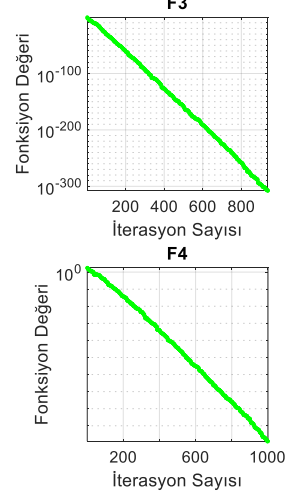

F5

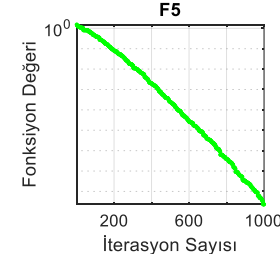

F6

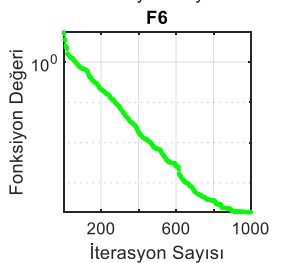

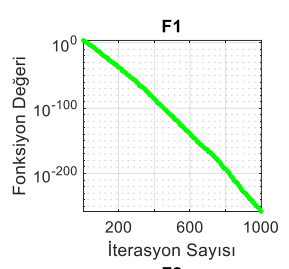

F2

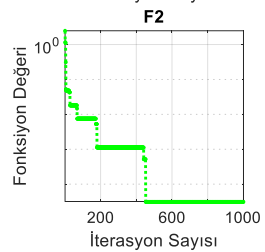

F3
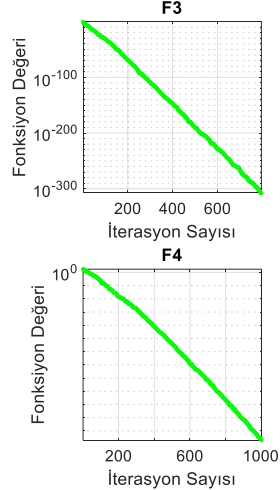

F5

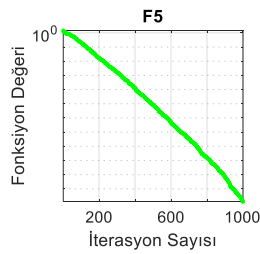

F6

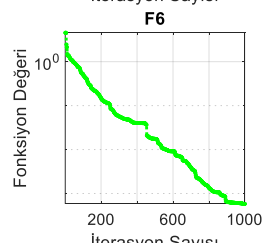

Iterasyon Sayıs|
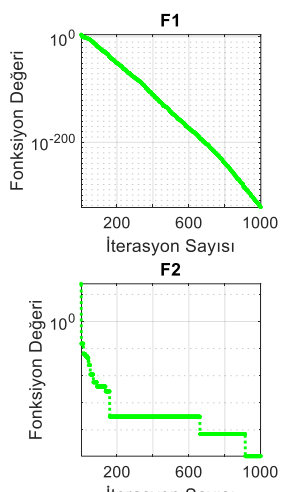

F3
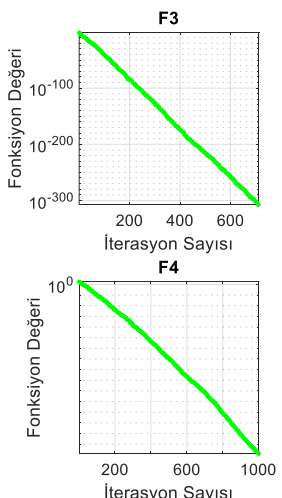

Iterasyon Sayısı

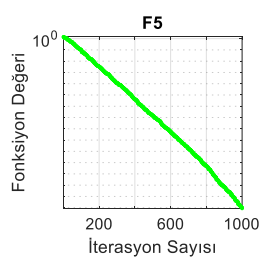

$\mathrm{F} 6$

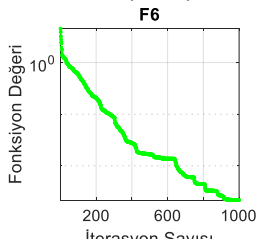

Iterasyon Sayısı

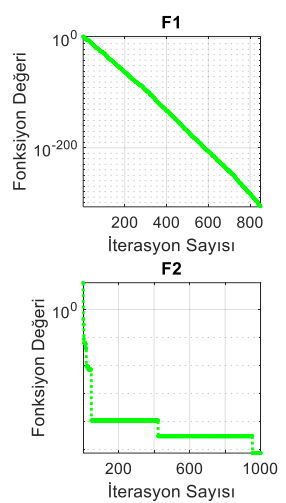

F3

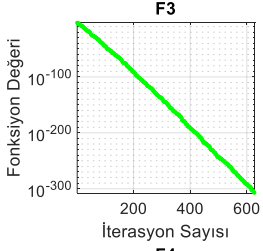

F4
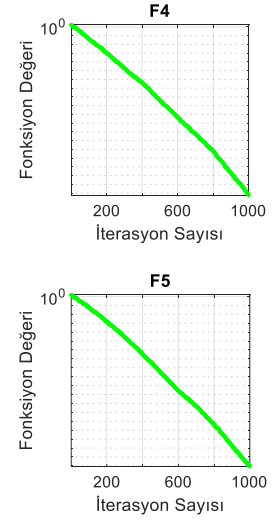

F6

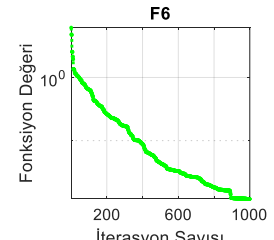


European Journal of Science and Technology
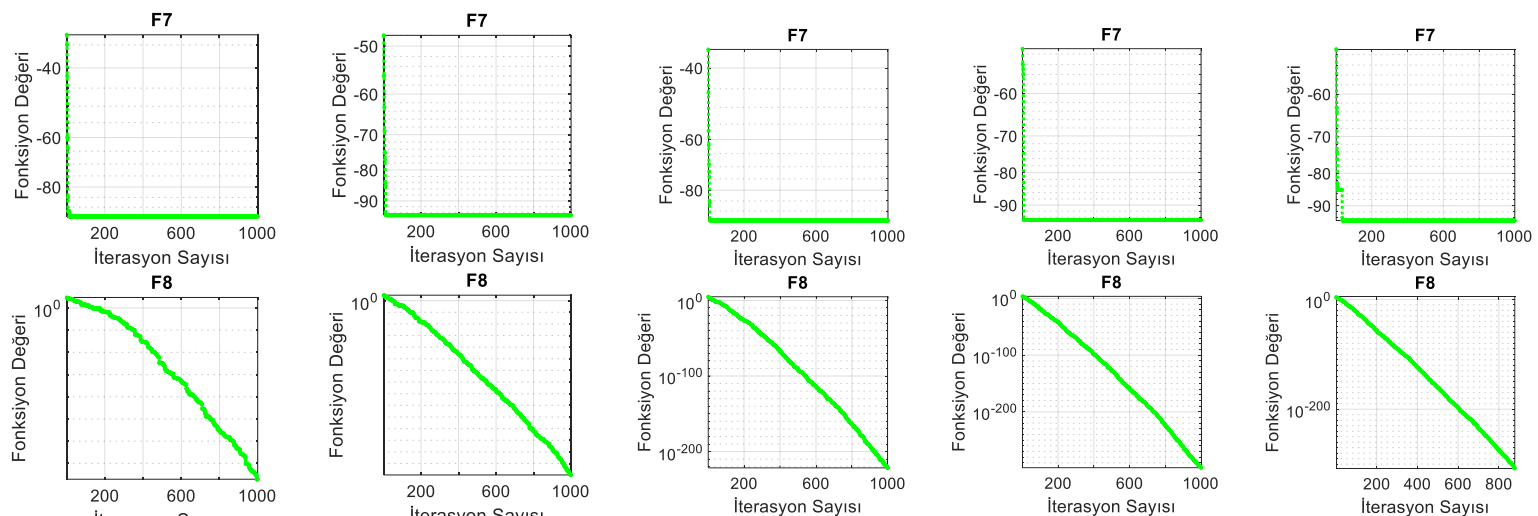

F8

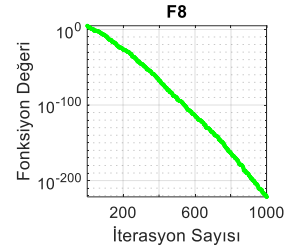

F8

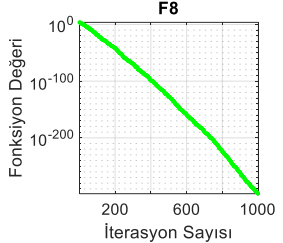

F8

F9

F9
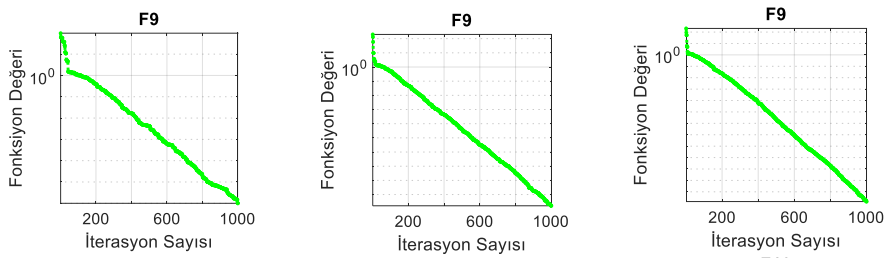

F9
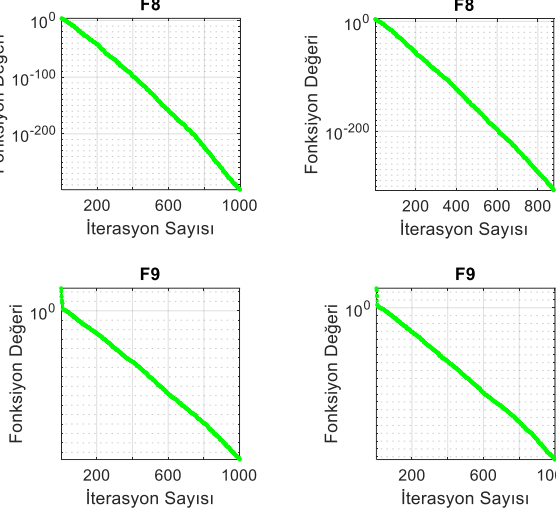

F10
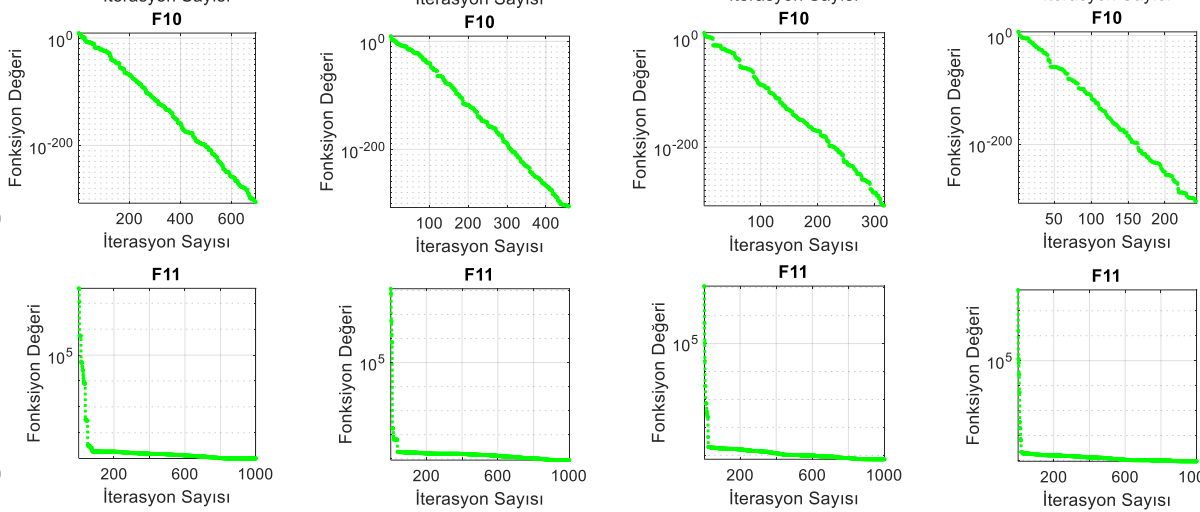

Iterasyon Sayıs
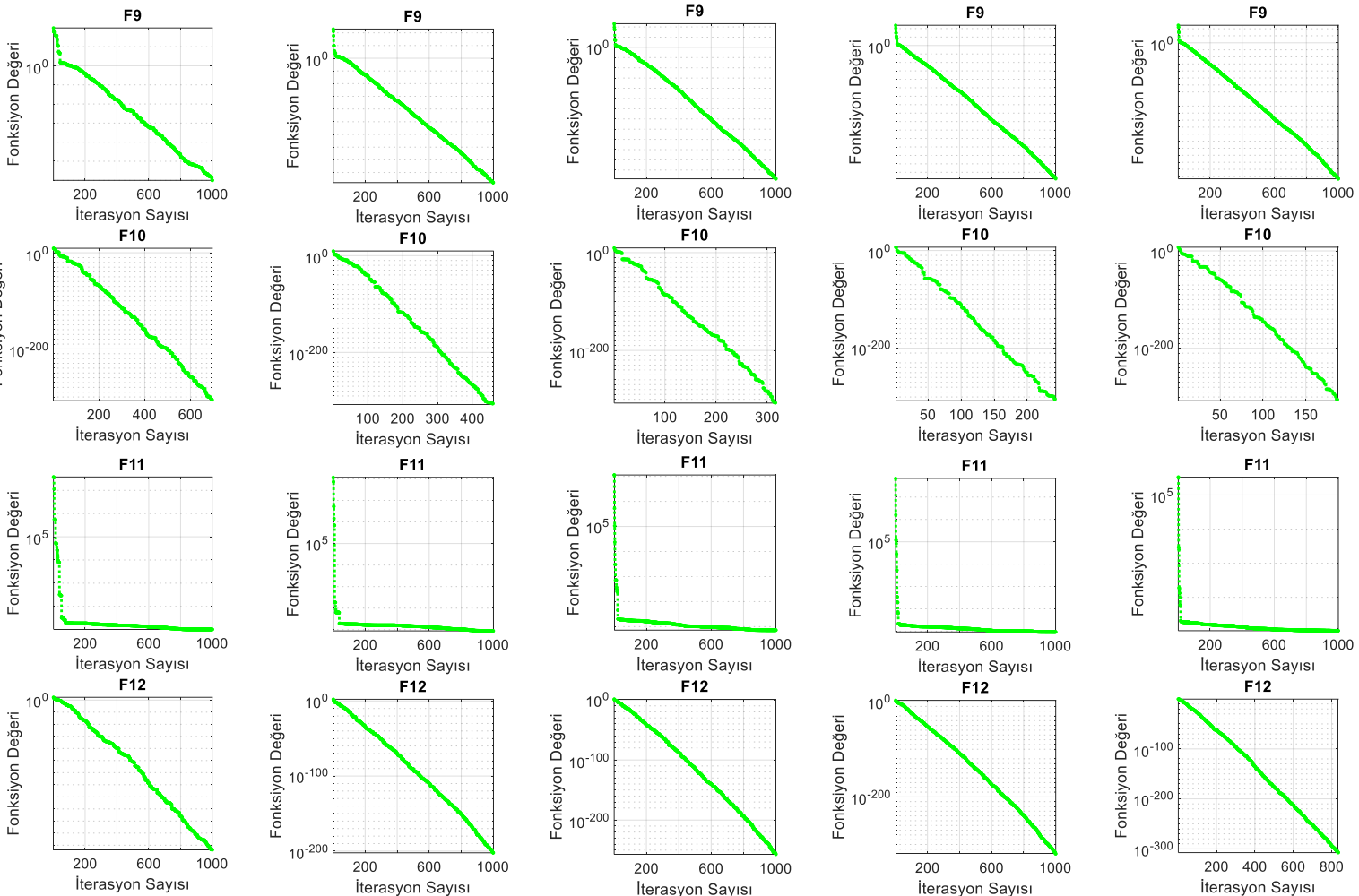

Sekil 3. Tek modlu fonksiyonlar (vektör saylst:25)

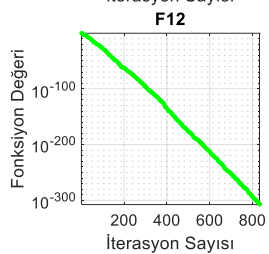

Tablo 6. Çok modlu fonksiyonlar (vektör sayısl:25)

\begin{tabular}{|c|c|c|c|c|c|c|}
\hline & & 0.1 & 0.3 & 0.5 & 0.7 & 0.9 \\
\hline \multirow{3}{*}{$F 1$} & En iyi & $4.54 \mathrm{E}+01$ & $3.57 \mathrm{E}+01$ & $5.55 \mathrm{E}+01$ & $3.45 \mathrm{E}+01$ & $7.91 \mathrm{E}+01$ \\
\hline & Ortalama & $1.08 \mathrm{E}+02$ & $1.08 \mathrm{E}+02$ & $1.15 \mathrm{E}+02$ & $1.17 \mathrm{E}+02$ & $1.32 \mathrm{E}+02$ \\
\hline & Std. & $3.13 \mathrm{E}+01$ & $2.95 \mathrm{E}+01$ & $2.78 \mathrm{E}+01$ & $2.97 \mathrm{E}+01$ & $2.66 \mathrm{E}+01$ \\
\hline \multirow{3}{*}{$F 2$} & En iyi & $0.00 \mathrm{E}+00$ & $0.00 \mathrm{E}+00$ & $0.00 \mathrm{E}+00$ & $0.00 \mathrm{E}+00$ & $0.00 \mathrm{E}+00$ \\
\hline & Ortalama & $0.00 \mathrm{E}+00$ & $0.00 \mathrm{E}+00$ & $0.00 \mathrm{E}+00$ & $0.00 \mathrm{E}+00$ & $0.00 \mathrm{E}+00$ \\
\hline & Std. & $0.00 \mathrm{E}+00$ & $0.00 \mathrm{E}+00$ & $0.00 \mathrm{E}+00$ & $0.00 \mathrm{E}+00$ & $0.00 \mathrm{E}+00$ \\
\hline \multirow{3}{*}{$F 3$} & En iyi & $9.00 \mathrm{E}-01$ & $9.00 \mathrm{E}-01$ & $9.00 \mathrm{E}-01$ & $9.00 \mathrm{E}-01$ & $9.00 \mathrm{E}-01$ \\
\hline & Ortalama & $9.47 \mathrm{E}-01$ & $9.03 \mathrm{E}-01$ & $9.00 \mathrm{E}-01$ & $9.00 \mathrm{E}-01$ & $9.00 \mathrm{E}-01$ \\
\hline & Std. & $5.00 \mathrm{E}-02$ & $1.80 \mathrm{E}-02$ & $4.44 \mathrm{E}-16$ & $4.44 \mathrm{E}-16$ & $4.44 \mathrm{E}-16$ \\
\hline \multirow{3}{*}{ F4 } & En iyi & $1.41 \mathrm{E}-16$ & $2.92 \mathrm{E}-15$ & $6.05 \mathrm{E}-14$ & $7.04 \mathrm{E}-11$ & $2.12 \mathrm{E}-07$ \\
\hline & Ortalama & $1.97 \mathrm{E}-10$ & $3.47 \mathrm{E}-12$ & $2.08 \mathrm{E}-10$ & $4.58 \mathrm{E}-07$ & $1.02 \mathrm{E}-04$ \\
\hline & Std. & $7.62 \mathrm{E}-10$ & $1.45 \mathrm{E}-11$ & $7.10 \mathrm{E}-10$ & $1.88 \mathrm{E}-06$ & $1.91 \mathrm{E}-04$ \\
\hline \multirow{3}{*}{$F 5$} & En iyi & $1.00 \mathrm{E}-60$ & $2.32 \mathrm{E}-100$ & $1.20 \mathrm{E}-128$ & $6.36 \mathrm{E}-165$ & $5.43 \mathrm{E}-198$ \\
\hline & Ortalama & $1.25 \mathrm{E}-05$ & $1.01 \mathrm{E}-91$ & $4.42 \mathrm{E}-120$ & $1.14 \mathrm{E}-151$ & $4.62 \mathrm{E}-182$ \\
\hline & Std. & $3.16 \mathrm{E}-05$ & $5.43 \mathrm{E}-91$ & $2.19 \mathrm{E}-119$ & 5.86E-151 & $0.00 \mathrm{E}+00$ \\
\hline \multirow{2}{*}{ F6 } & En iyi & $3.28 \mathrm{E}-40$ & $7.58 \mathrm{E}-82$ & $1.37 \mathrm{E}-110$ & $1.93 \mathrm{E}-128$ & $7.04 \mathrm{E}-139$ \\
\hline & Ortalama & 3.93E-19 & 7.98E-16 & $1.14 \mathrm{E}-55$ & $1.17 \mathrm{E}-66$ & $7.41 \mathrm{E}-51$ \\
\hline
\end{tabular}


Avrupa Bilim ve Teknoloji Dergisi

\begin{tabular}{c|l|l|l|l|l|l}
\hline & Std. & $2.12 \mathrm{E}-18$ & $4.30 \mathrm{E}-15$ & $6.13 \mathrm{E}-55$ & $6.31 \mathrm{E}-66$ & $3.99 \mathrm{E}-50$ \\
\hline \multirow{5}{*}{$F 7$} & En iyi & $0.00 \mathrm{E}+00$ & $0.00 \mathrm{E}+00$ & $0.00 \mathrm{E}+00$ & $0.00 \mathrm{E}+00$ & $0.00 \mathrm{E}+00$ \\
\cline { 2 - 7 } & Ortalama & $0.00 \mathrm{E}+00$ & $0.00 \mathrm{E}+00$ & $0.00 \mathrm{E}+00$ & $0.00 \mathrm{E}+00$ & $0.00 \mathrm{E}+00$ \\
\cline { 2 - 7 } & Std. & $0.00 \mathrm{E}+00$ & $0.00 \mathrm{E}+00$ & $0.00 \mathrm{E}+00$ & $0.00 \mathrm{E}+00$ & $0.00 \mathrm{E}+00$ \\
\hline \multirow{5}{*}{$F 8$} & En iyi & $\mathbf{1 . 0 0 E}+\mathbf{0 0}$ & $1.00 \mathrm{E}+00$ & $1.90 \mathrm{E}+00$ & $5.03 \mathrm{E}+00$ & $6.38 \mathrm{E}+00$ \\
\cline { 2 - 7 } & Ortalama & $4.49 \mathrm{E}+00$ & $8.01 \mathrm{E}+00$ & $1.24 \mathrm{E}+01$ & $1.67 \mathrm{E}+01$ & $1.93 \mathrm{E}+01$ \\
\cline { 2 - 7 } & Std. & $3.15 \mathrm{E}+00$ & $3.52 \mathrm{E}+00$ & $7.03 \mathrm{E}+00$ & $6.46 \mathrm{E}+00$ & $8.72 \mathrm{E}+00$ \\
\hline \multirow{5}{*}{$F 9$} & En iyi & $5.35 \mathrm{E}-38$ & $2.65 \mathrm{E}-88$ & $1.07 \mathrm{E}-118$ & $1.95 \mathrm{E}-149$ & $\mathbf{0 . 0 0 E}+\mathbf{0 0}$ \\
\cline { 2 - 7 } & Ortalama & $3.03 \mathrm{E}-02$ & $2.78 \mathrm{E}-12$ & $6.16 \mathrm{E}-81$ & $1.96 \mathrm{E}-120$ & $5.46 \mathrm{E}-160$ \\
\cline { 2 - 7 } & Std. & $4.56 \mathrm{E}-02$ & $1.50 \mathrm{E}-11$ & $3.32 \mathrm{E}-80$ & $1.05 \mathrm{E}-119$ & $2.94 \mathrm{E}-159$ \\
\hline \multirow{5}{*}{$F 10$} & En iyi & $-\mathbf{7 . 8 3 E}+\mathbf{0 2}$ & $-7.55 \mathrm{E}+02$ & $-7.55 \mathrm{E}+02$ & $-7.27 \mathrm{E}+02$ & $-7.69 \mathrm{E}+02$ \\
\cline { 2 - 7 } & Ortalama & $-7.35 \mathrm{E}+02$ & $-7.11 \mathrm{E}+02$ & $-6.93 \mathrm{E}+02$ & $-6.90 \mathrm{E}+02$ & $-6.87 \mathrm{E}+02$ \\
\cline { 2 - 7 } & Std. & $3.47 \mathrm{E}+01$ & $2.02 \mathrm{E}+01$ & $2.95 \mathrm{E}+01$ & $2.82 \mathrm{E}+01$ & $3.35 \mathrm{E}+01$ \\
\hline \multirow{3}{*}{$F 12$} & En iyi & $0.00 \mathrm{E}+00$ & $0.00 \mathrm{E}+00$ & $0.00 \mathrm{E}+00$ & $0.00 \mathrm{E}+00$ & $0.00 \mathrm{E}+00$ \\
\cline { 2 - 7 } & Ortalama & $0.00 \mathrm{E}+00$ & $0.00 \mathrm{E}+00$ & $0.00 \mathrm{E}+00$ & $0.00 \mathrm{E}+00$ & $0.00 \mathrm{E}+00$ \\
\cline { 2 - 7 } & Std. & $0.00 \mathrm{E}+00$ & $0.00 \mathrm{E}+00$ & $0.00 \mathrm{E}+00$ & $0.00 \mathrm{E}+00$ & $0.00 \mathrm{E}+00$ \\
\hline & En iyi & $-1.00 \mathrm{E}+00$ & $-1.00 \mathrm{E}+00$ & $-1.00 \mathrm{E}+00$ & $-1.00 \mathrm{E}+00$ & $-1.00 \mathrm{E}+00$ \\
\cline { 2 - 7 } & Ortalama & $-1.00 \mathrm{E}+00$ & $-1.00 \mathrm{E}+00$ & $-1.00 \mathrm{E}+00$ & $-1.00 \mathrm{E}+00$ & $-1.00 \mathrm{E}+00$ \\
\cline { 2 - 7 } & Std. & $1.85 \mathrm{E}-13$ & $0.00 \mathrm{E}+00$ & $0.00 \mathrm{E}+00$ & $0.00 \mathrm{E}+00$ & $0.00 \mathrm{E}+00$ \\
\hline
\end{tabular}
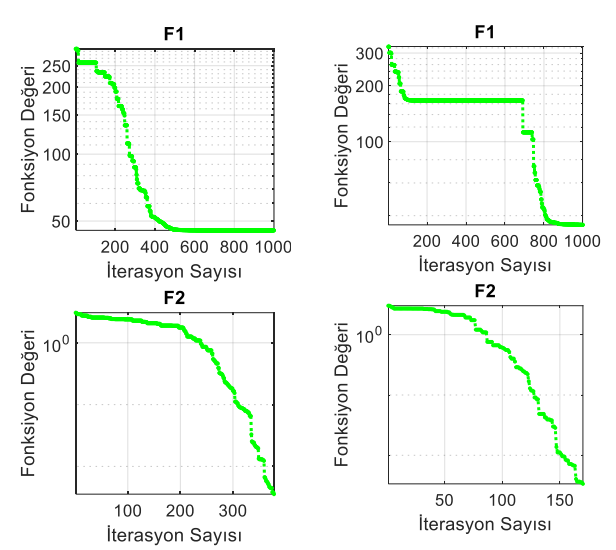

F3

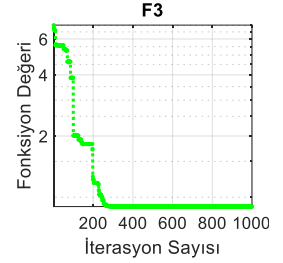

F4

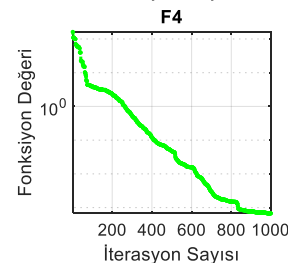

F5
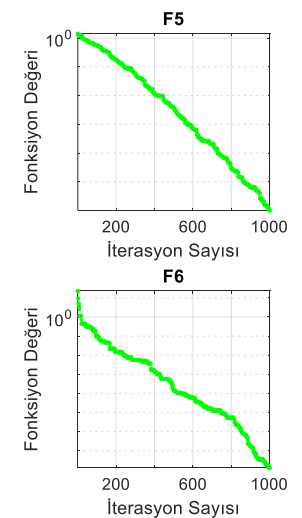

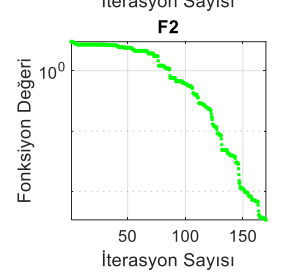

F3

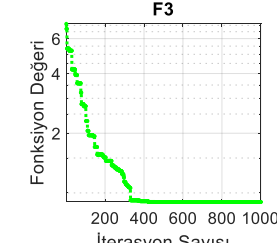

F4

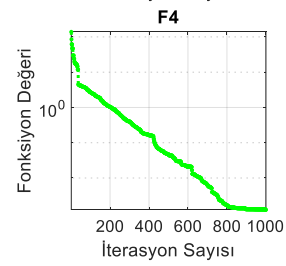

F5

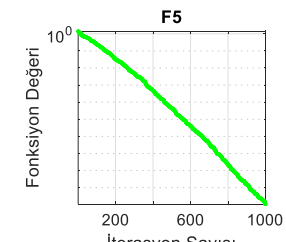

F6

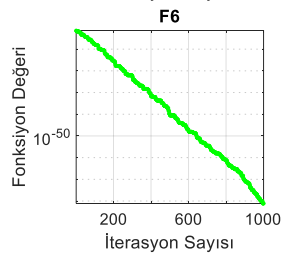

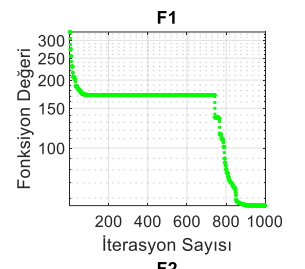
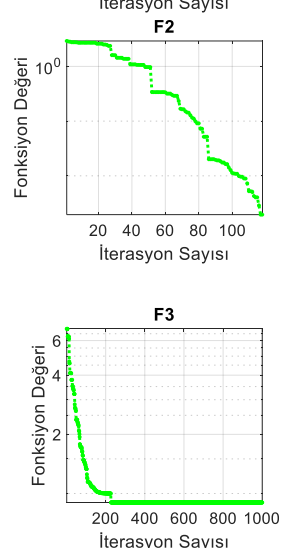

F4

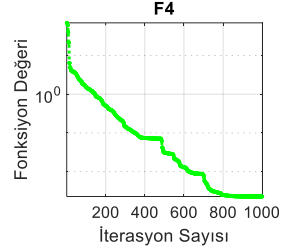

F5

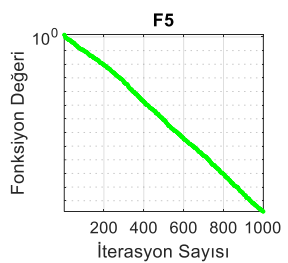

F6

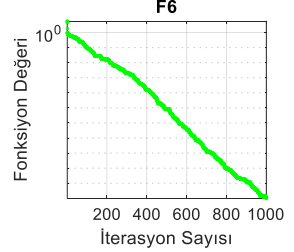

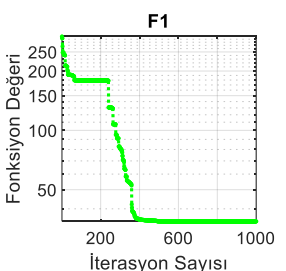

F2
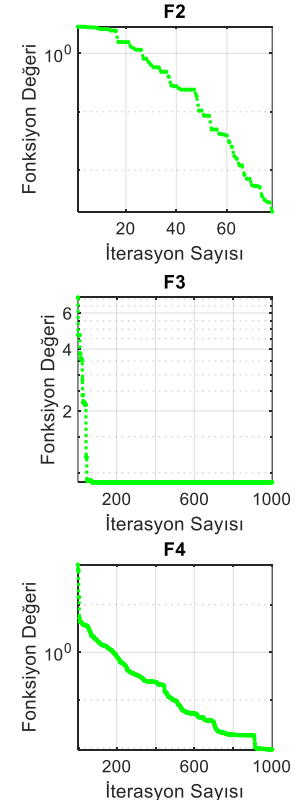

F5

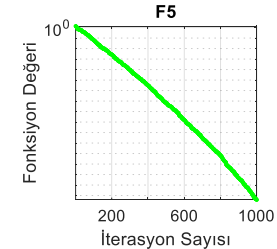

F6

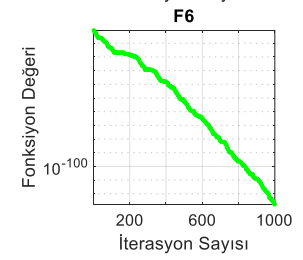

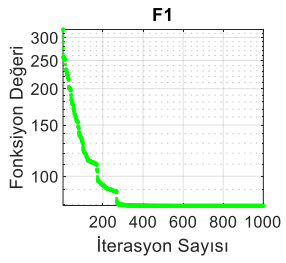

F2
F

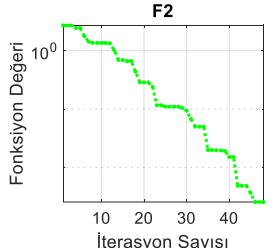

Iterasyon Sayıs!

F3

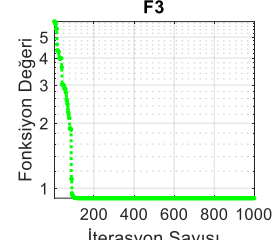

F4

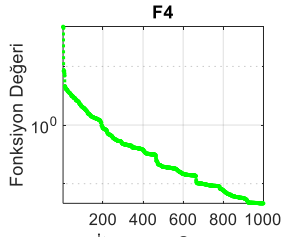

200400600800
Iterasyon Sayıs!

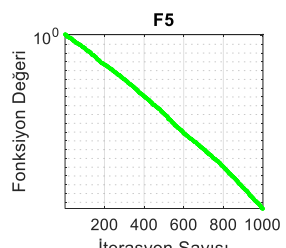

Iterasyon Sayısı

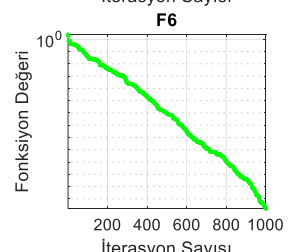

$200400600 \quad 800100$ 
European Journal of Science and Technology
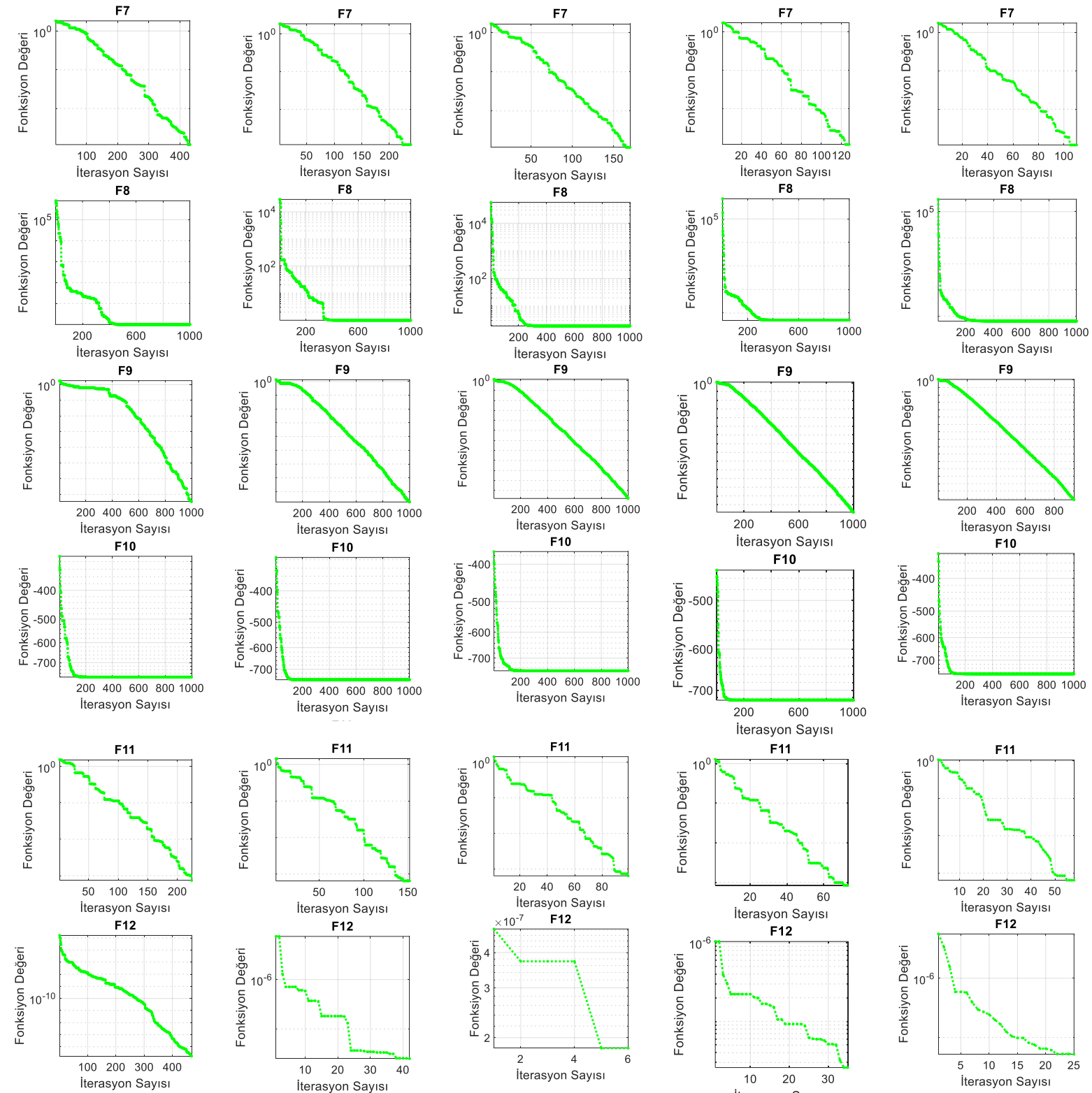

Şekil 4. Çok modlu fonksiyonlar (vektör saylsı:25)

Tablo 7. Tek modlu fonksiyonlar (vektör sayısı:50)

\begin{tabular}{|c|c|c|c|c|c|c|}
\hline & & 0.1 & 0.3 & 0.5 & 0.7 & 0.9 \\
\hline \multirow{3}{*}{$F 1$} & En iyi & $2.02 \mathrm{E}-149$ & $5.84 \mathrm{E}-224$ & $1.51 \mathrm{E}-285$ & $0.00 \mathrm{E}+00$ & $0.00 \mathrm{E}+00$ \\
\hline & Ortalama & $5.78 \mathrm{E}-134$ & $9.53 \mathrm{E}-212$ & $1.41 \mathrm{E}-271$ & $0.00 \mathrm{E}+00$ & $0.00 \mathrm{E}+00$ \\
\hline & Std. & $3.11 \mathrm{E}-133$ & $0.00 \mathrm{E}+00$ & $0.00 \mathrm{E}+00$ & $0.00 \mathrm{E}+00$ & $0.00 \mathrm{E}+00$ \\
\hline \multirow{3}{*}{$F 2$} & En iyi & $7.58 \mathrm{E}-05$ & $4.66 \mathrm{E}-05$ & $3.34 \mathrm{E}-05$ & $1.12 \mathrm{E}-05$ & $7.37 \mathrm{E}-06$ \\
\hline & Ortalama & $6.20 \mathrm{E}-04$ & $3.16 \mathrm{E}-04$ & $2.43 \mathrm{E}-04$ & $1.87 \mathrm{E}-04$ & $1.94 \mathrm{E}-04$ \\
\hline & Std. & $3.60 \mathrm{E}-04$ & $1.58 \mathrm{E}-04$ & $1.79 \mathrm{E}-04$ & $1.32 \mathrm{E}-04$ & $2.12 \mathrm{E}-04$ \\
\hline \multirow{3}{*}{$F 3$} & En iyi & $4.67 \mathrm{E}-274$ & $0.00 \mathrm{E}+00$ & $0.00 \mathrm{E}+00$ & $0.00 \mathrm{E}+00$ & $0.00 \mathrm{E}+00$ \\
\hline & Ortalama & $2.93 \mathrm{E}-252$ & $0.00 \mathrm{E}+00$ & $0.00 \mathrm{E}+00$ & $0.00 \mathrm{E}+00$ & $0.00 \mathrm{E}+00$ \\
\hline & Std. & $0.00 \mathrm{E}+00$ & $0.00 \mathrm{E}+00$ & $0.00 \mathrm{E}+00$ & $0.00 \mathrm{E}+00$ & $0.00 \mathrm{E}+00$ \\
\hline \multirow{3}{*}{ F4 } & En iyi & $3.11 \mathrm{E}-78$ & $5.97 \mathrm{E}-113$ & $4.47 \mathrm{E}-144$ & $6.46 \mathrm{E}-174$ & $1.26 \mathrm{E}-209$ \\
\hline & Ortalama & $3.97 \mathrm{E}-71$ & $3.74 \mathrm{E}-108$ & $2.54 \mathrm{E}-137$ & $8.97 \mathrm{E}-168$ & $1.73 \mathrm{E}-203$ \\
\hline & Std. & $8.48 \mathrm{E}-71$ & $1.36 \mathrm{E}-107$ & $1.09 \mathrm{E}-136$ & $0.00 \mathrm{E}+00$ & $0.00 \mathrm{E}+00$ \\
\hline \multirow{3}{*}{$F 5$} & En iyi & $3.82 \mathrm{E}-60$ & $9.95 \mathrm{E}-99$ & $1.70 \mathrm{E}-131$ & $3.10 \mathrm{E}-168$ & $1.10 \mathrm{E}-208$ \\
\hline & Ortalama & $5.76 \mathrm{E}-53$ & $2.01 \mathrm{E}-92$ & $5.75 \mathrm{E}-127$ & $3.11 \mathrm{E}-162$ & $4.08 \mathrm{E}-199$ \\
\hline & Std. & $3.01 \mathrm{E}-52$ & $6.40 \mathrm{E}-92$ & $1.29 \mathrm{E}-126$ & $1.37 \mathrm{E}-161$ & $0.00 \mathrm{E}+00$ \\
\hline F6 & En iyi & $7.94 \mathrm{E}-30$ & $1.29 \mathrm{E}-30$ & $3.22 \mathrm{E}-30$ & $1.05 \mathrm{E}-25$ & $6.18 \mathrm{E}-18$ \\
\hline
\end{tabular}


Avrupa Bilim ve Teknoloji Dergisi

\begin{tabular}{c|l|l|l|l|l|l}
\hline \multirow{5}{*}{$F$} & Ortalama & $2.33 \mathrm{E}-26$ & $7.77 \mathrm{E}-28$ & $6.77 \mathrm{E}-26$ & $5.21 \mathrm{E}-22$ & $7.31 \mathrm{E}-12$ \\
\cline { 2 - 7 } & Std. & $5.63 \mathrm{E}-26$ & $1.73 \mathrm{E}-27$ & $2.15 \mathrm{E}-25$ & $1.38 \mathrm{E}-21$ & $3.93 \mathrm{E}-11$ \\
\hline \multirow{5}{*}{$F 7$} & En iyi & $-9.50 \mathrm{E}+01$ & $-9.50 \mathrm{E}+01$ & $-9.50 \mathrm{E}+01$ & $-9.50 \mathrm{E}+01$ & $-9.50 \mathrm{E}+01$ \\
\cline { 2 - 7 } & Ortalama & $-9.50 \mathrm{E}+01$ & $-9.50 \mathrm{E}+01$ & $-9.50 \mathrm{E}+01$ & $-9.50 \mathrm{E}+01$ & $-9.50 \mathrm{E}+01$ \\
\cline { 2 - 7 } & Std. & $0.00 \mathrm{E}+00$ & $0.00 \mathrm{E}+00$ & $0.00 \mathrm{E}+00$ & $0.00 \mathrm{E}+00$ & $0.00 \mathrm{E}+00$ \\
\hline \multirow{5}{*}{$F 9$} & En iyi & $1.04 \mathrm{E}-92$ & $5.58 \mathrm{E}-169$ & $1.18 \mathrm{E}-245$ & $4.00 \mathrm{E}-323$ & $\mathbf{0 . 0 0 E}+\mathbf{0 0}$ \\
\cline { 2 - 7 } & Ortalama & $1.10 \mathrm{E}-78$ & $1.08 \mathrm{E}-157$ & $3.29 \mathrm{E}-226$ & $1.79 \mathrm{E}-304$ & $0.00 \mathrm{E}+00$ \\
\cline { 2 - 7 } & Std. & $4.21 \mathrm{E}-78$ & $3.17 \mathrm{E}-157$ & $0.00 \mathrm{E}+00$ & $0.00 \mathrm{E}+00$ & $0.00 \mathrm{E}+00$ \\
\hline \multirow{5}{*}{$F 10$} & En iyi & $1.57 \mathrm{E}-76$ & $4.36 \mathrm{E}-111$ & $1.74 \mathrm{E}-143$ & $1.65 \mathrm{E}-177$ & $\mathbf{4 . 5 3 E - 2 1 0}$ \\
\cline { 2 - 7 } & Ortalama & $3.89 \mathrm{E}-68$ & $1.81 \mathrm{E}-107$ & $7.73 \mathrm{E}-138$ & $1.21 \mathrm{E}-166$ & $8.26 \mathrm{E}-202$ \\
\cline { 2 - 7 } & Std. & $1.83 \mathrm{E}-67$ & $7.16 \mathrm{E}-107$ & $1.81 \mathrm{E}-137$ & $0.00 \mathrm{E}+00$ & $0.00 \mathrm{E}+00$ \\
\cline { 2 - 7 } & En iyi & $0.00 \mathrm{E}+00$ & $0.00 \mathrm{E}+00$ & $0.00 \mathrm{E}+00$ & $0.00 \mathrm{E}+00$ & $0.00 \mathrm{E}+00$ \\
\cline { 2 - 7 } & Ortalama & $0.00 \mathrm{E}+00$ & $0.00 \mathrm{E}+00$ & $0.00 \mathrm{E}+00$ & $0.00 \mathrm{E}+00$ & $0.00 \mathrm{E}+00$ \\
\cline { 2 - 7 } & Std. & $0.00 \mathrm{E}+00$ & $0.00 \mathrm{E}+00$ & $0.00 \mathrm{E}+00$ & $0.00 \mathrm{E}+00$ & $0.00 \mathrm{E}+00$ \\
\hline \multirow{3}{*}{$F 11$} & En iyi & $6.28 \mathrm{E}+00$ & $1.27 \mathrm{E}+00$ & $1.23 \mathrm{E}+00$ & $\mathbf{4 . 9 2 E - 0 1}$ & $1.06 \mathrm{E}+00$ \\
\cline { 2 - 7 } & Ortalama & $8.79 \mathrm{E}+00$ & $5.26 \mathrm{E}+00$ & $3.67 \mathrm{E}+00$ & $4.29 \mathrm{E}+00$ & $5.66 \mathrm{E}+00$ \\
\cline { 2 - 7 } & Std. & $1.44 \mathrm{E}+00$ & $1.80 \mathrm{E}+00$ & $1.88 \mathrm{E}+00$ & $2.52 \mathrm{E}+00$ & $1.65 \mathrm{E}+00$ \\
\hline & En iyi & $2.45 \mathrm{E}-159$ & $2.96 \mathrm{E}-235$ & $1.47 \mathrm{E}-286$ & $\mathbf{0 . 0 0 E}+\mathbf{0 0}$ & $\mathbf{0 . 0 0 E}+\mathbf{0 0}$ \\
\cline { 2 - 7 } & Ortalama & $3.86 \mathrm{E}-145$ & $8.92 \mathrm{E}-219$ & $6.18 \mathrm{E}-275$ & $0.00 \mathrm{E}+00$ & $0.00 \mathrm{E}+00$ \\
\cline { 2 - 7 } & Std. & $1.26 \mathrm{E}-144$ & $0.00 \mathrm{E}+00$ & $0.00 \mathrm{E}+00$ & $0.00 \mathrm{E}+00$ & $0.00 \mathrm{E}+00$ \\
\hline
\end{tabular}

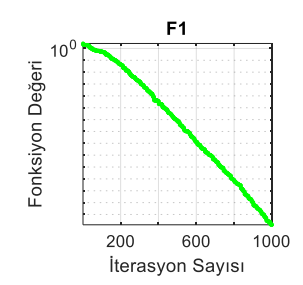

F2
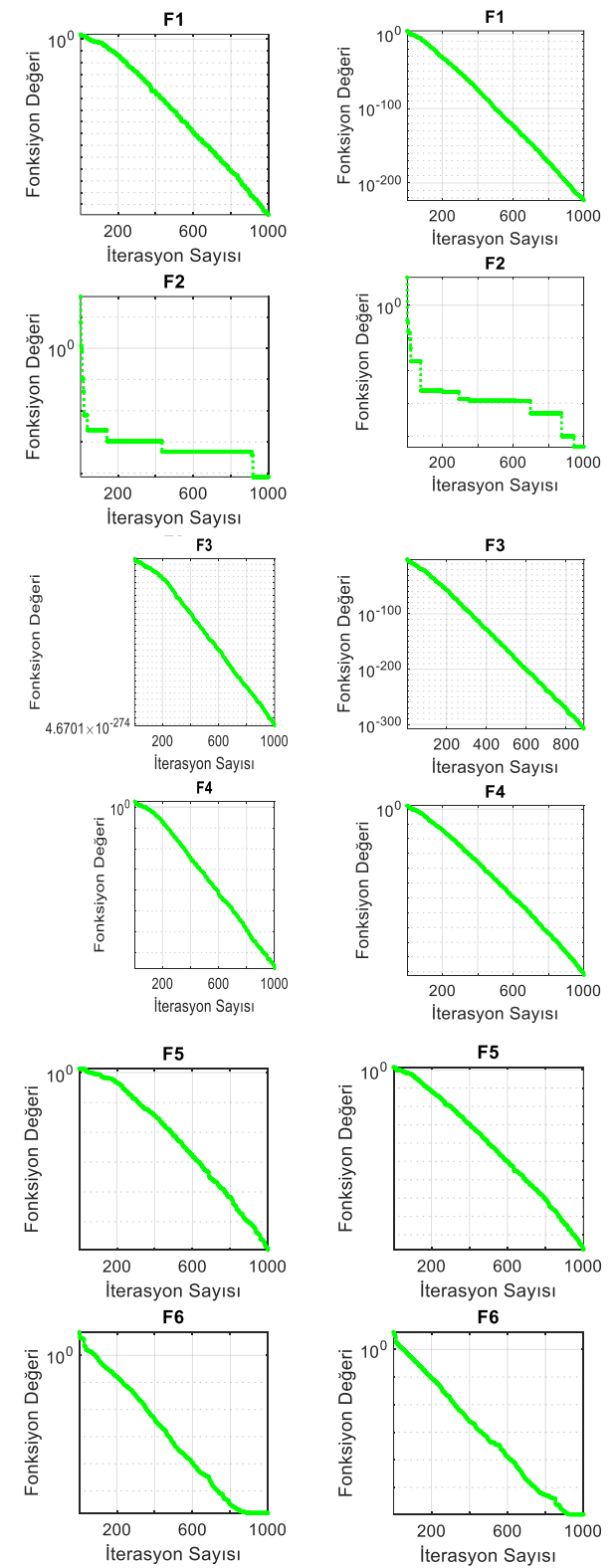

F2
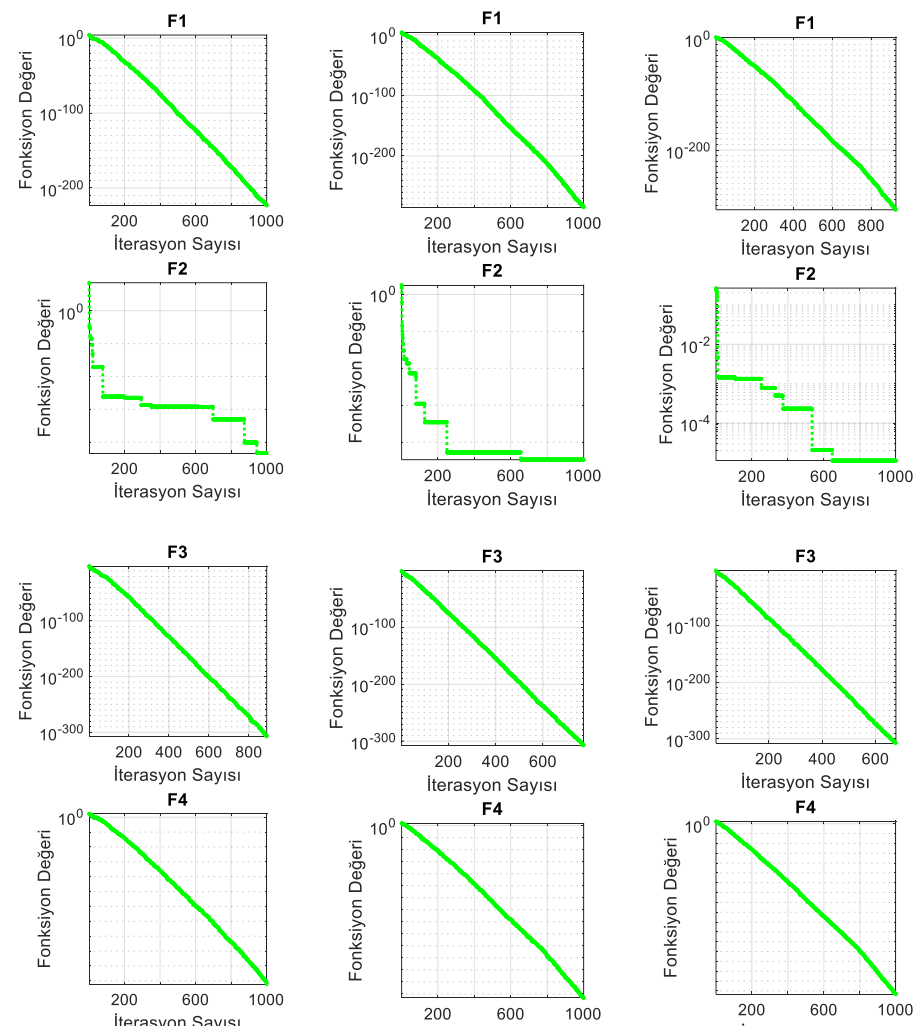

F5
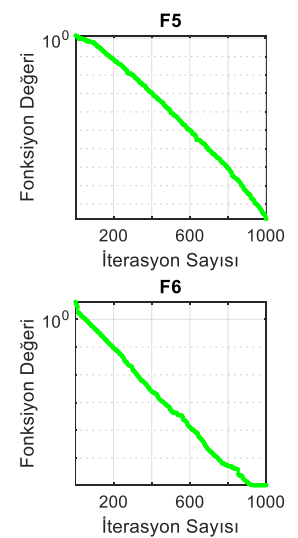
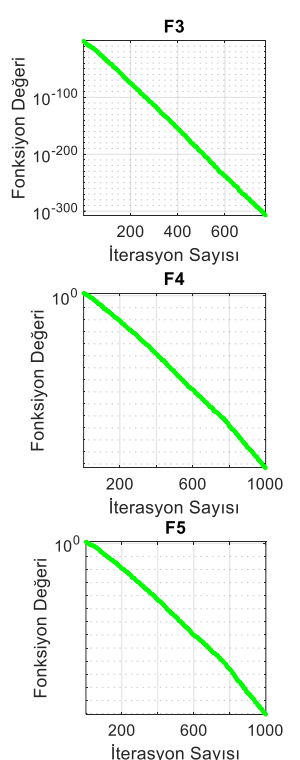

F6

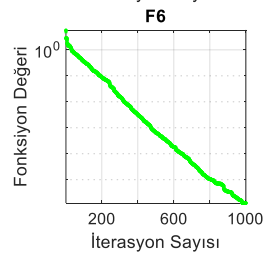

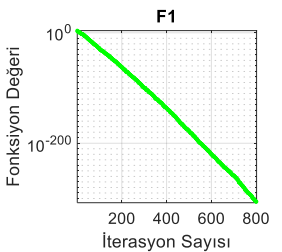
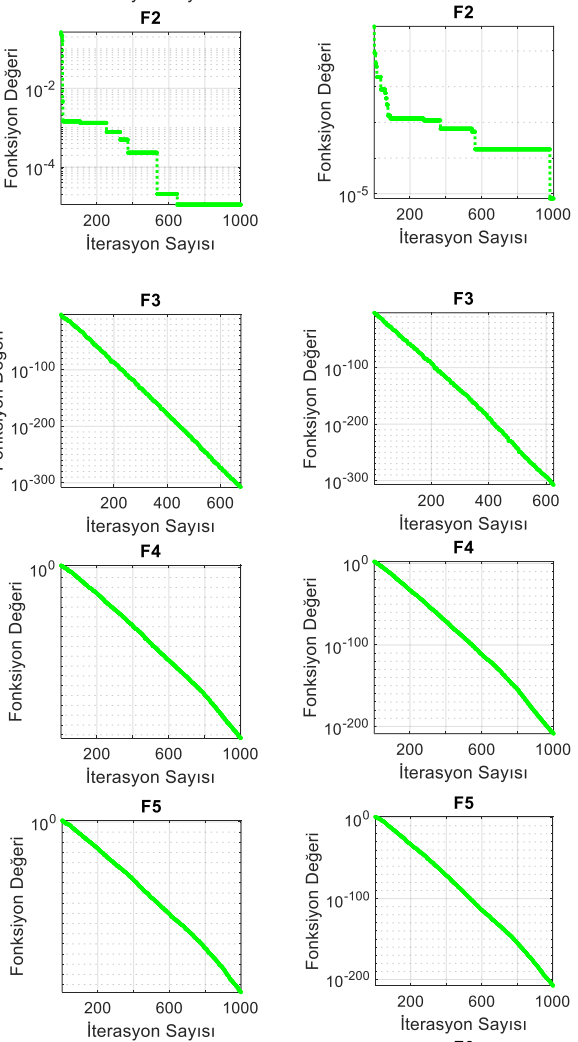

F6
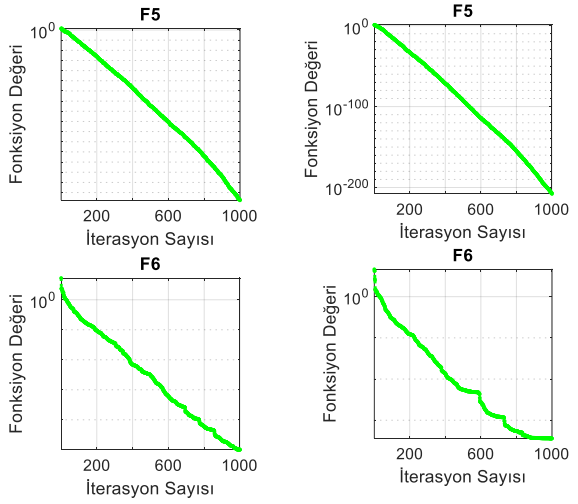
European Journal of Science and Technology
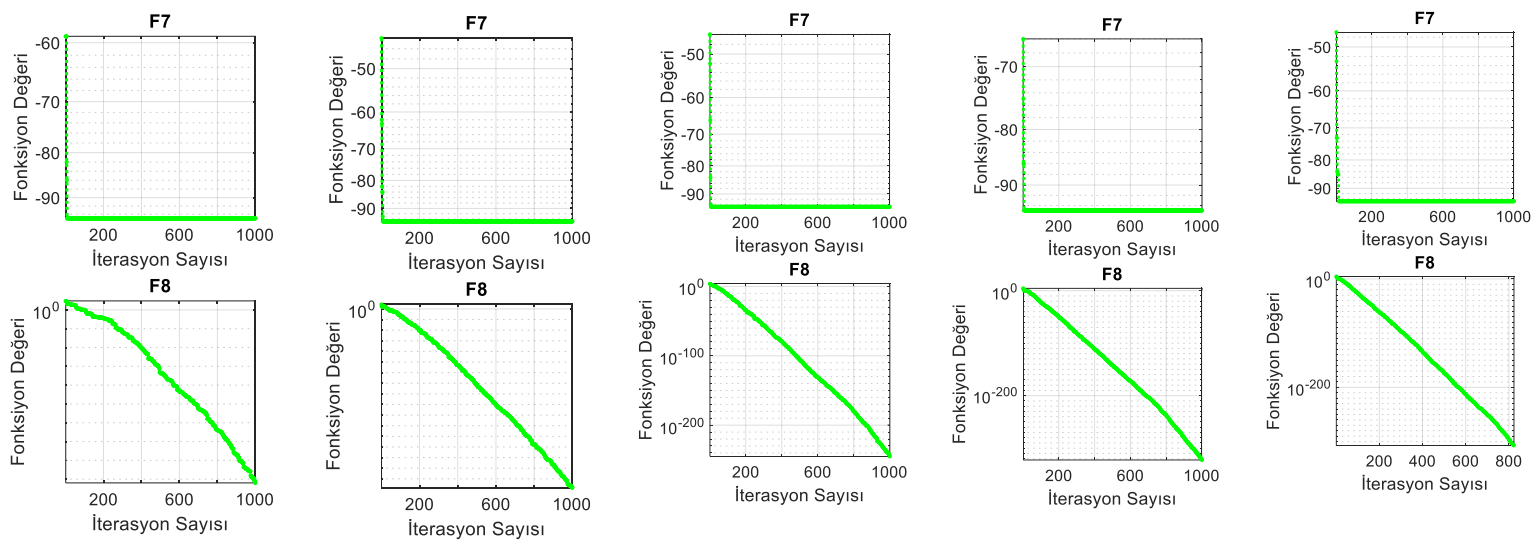

F9
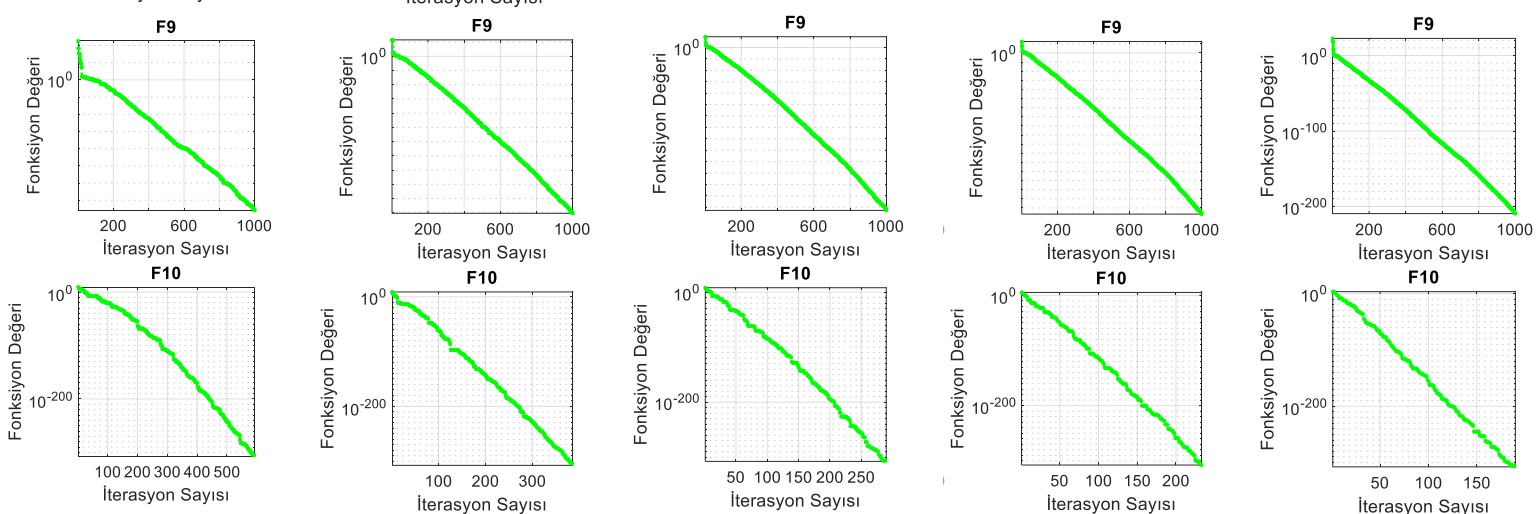

F11
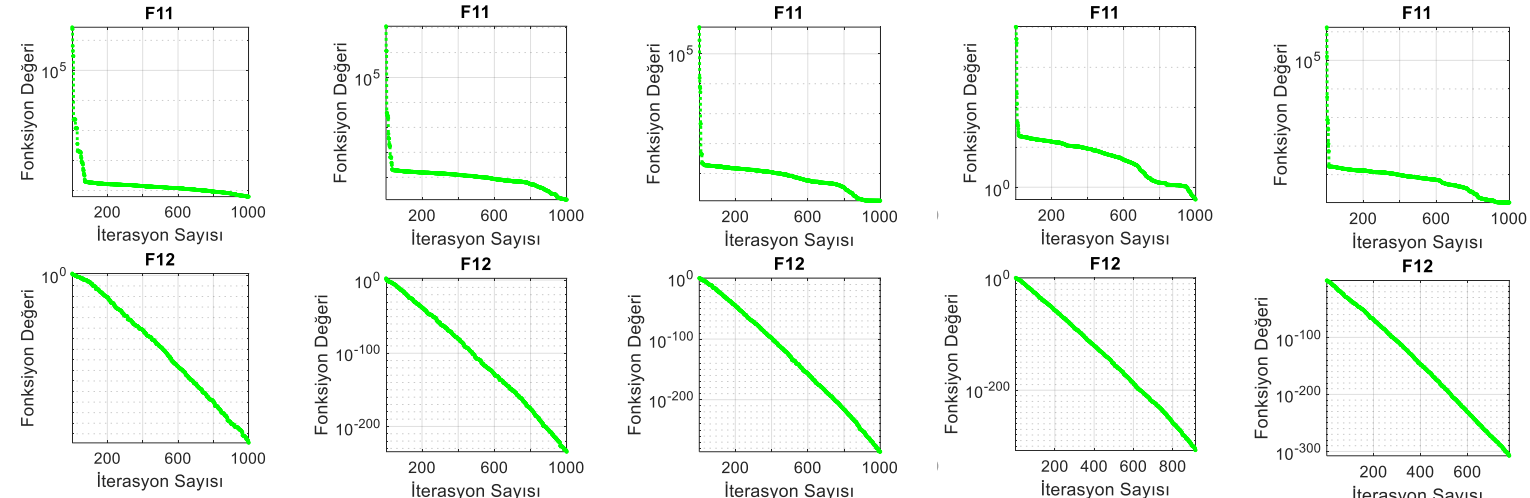

F12

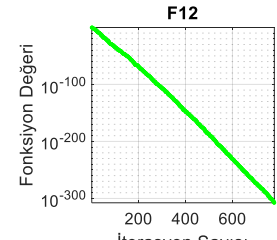

Sekil 5. Tek modlu fonksiyonlar (vektör saylsı:50)

Tablo 8. Çok modlu fonksiyonlar (vektör saylsı:50)

\begin{tabular}{c|l|l|l|l|l|l}
\hline & & $\mathbf{0 . 1}$ & $\mathbf{0 . 3}$ & $\mathbf{0 . 5}$ & $\mathbf{0 . 7}$ & $\mathbf{0 . 9}$ \\
\hline \multirow{3}{*}{$F 1$} & En iyi & $\mathbf{5 . 9 2 E}+\mathbf{0 0}$ & $2.37 \mathrm{E}+01$ & $2.37 \mathrm{E}+01$ & $5.73 \mathrm{E}+01$ & $8.71 \mathrm{E}+01$ \\
\cline { 2 - 7 } & Ortalama & $8.98 \mathrm{E}+01$ & $9.74 \mathrm{E}+01$ & $1.05 \mathrm{E}+02$ & $1.11 \mathrm{E}+02$ & $1.27 \mathrm{E}+02$ \\
\cline { 2 - 7 } & Std. & $4.35 \mathrm{E}+01$ & $3.45 \mathrm{E}+01$ & $3.59 \mathrm{E}+01$ & $2.47 \mathrm{E}+01$ & $2.16 \mathrm{E}+01$ \\
\hline \multirow{3}{*}{$F 2$} & En iyi & $0.00 \mathrm{E}+00$ & $0.00 \mathrm{E}+00$ & $0.00 \mathrm{E}+00$ & $0.00 \mathrm{E}+00$ & $0.00 \mathrm{E}+00$ \\
\cline { 2 - 7 } & Ortalama & $0.00 \mathrm{E}+00$ & $0.00 \mathrm{E}+00$ & $0.00 \mathrm{E}+00$ & $0.00 \mathrm{E}+00$ & $0.00 \mathrm{E}+00$ \\
\cline { 2 - 7 } & Std. & $0.00 \mathrm{E}+00$ & $0.00 \mathrm{E}+00$ & $0.00 \mathrm{E}+00$ & $0.00 \mathrm{E}+00$ & $0.00 \mathrm{E}+00$ \\
\hline \multirow{5}{*}{$F 3$} & En iyi & $9.00 \mathrm{E}-01$ & $9.00 \mathrm{E}-01$ & $9.00 \mathrm{E}-01$ & $9.00 \mathrm{E}-01$ & $9.00 \mathrm{E}-01$ \\
\cline { 2 - 7 } & Ortalama & $9.13 \mathrm{E}-01$ & $9.00 \mathrm{E}-01$ & $9.00 \mathrm{E}-01$ & $9.00 \mathrm{E}-01$ & $9.00 \mathrm{E}-01$ \\
\cline { 2 - 7 } & Std. & $3.40 \mathrm{E}-02$ & $4.44 \mathrm{E}-16$ & $4.44 \mathrm{E}-16$ & $4.44 \mathrm{E}-16$ & $4.44 \mathrm{E}-16$ \\
\hline \multirow{3}{*}{$F 4$} & En iyi & $1.04 \mathrm{E}-24$ & $4.68 \mathrm{E}-27$ & $\mathbf{4 . 6 6 E}-26$ & $7.86 \mathrm{E}-22$ & $1.55 \mathrm{E}-14$ \\
\cline { 2 - 7 } & Ortalama & $3.61 \mathrm{E}-20$ & $1.80 \mathrm{E}-23$ & $1.78 \mathrm{E}-21$ & $9.10 \mathrm{E}-18$ & $8.25 \mathrm{E}-10$ \\
\cline { 2 - 7 } & Std. & $1.08 \mathrm{E}-19$ & $7.82 \mathrm{E}-23$ & $7.85 \mathrm{E}-21$ & $2.72 \mathrm{E}-17$ & $3.80 \mathrm{E}-09$ \\
\hline \multirow{3}{*}{$F 5$} & En iyi & $2.14 \mathrm{E}-81$ & $2.35 \mathrm{E}-114$ & $2.23 \mathrm{E}-145$ & $1.31 \mathrm{E}-176$ & $\mathbf{7 . 4 7 E}-213$ \\
\cline { 2 - 7 } & Ortalama & $6.30 \mathrm{E}-07$ & $1.51 \mathrm{E}-110$ & $3.28 \mathrm{E}-136$ & $7.19 \mathrm{E}-171$ & $9.56 \mathrm{E}-206$ \\
\cline { 2 - 7 } & Std. & $3.39 \mathrm{E}-06$ & $3.39 \mathrm{E}-110$ & $1.76 \mathrm{E}-135$ & $0.00 \mathrm{E}+00$ & $0.00 \mathrm{E}+00$ \\
\hline$F 6$ & En iyi & $1.96 \mathrm{E}-40$ & $2.91 \mathrm{E}-79$ & $6.81 \mathrm{E}-115$ & $2.93 \mathrm{E}-142$ & $\mathbf{9 . 7 5 E}-16 \mathbf{1 0}$ \\
\hline
\end{tabular}


Avrupa Bilim ve Teknoloji Dergisi

\begin{tabular}{c|l|l|l|l|l|l}
\hline \multirow{5}{*}{$F$} & Ortalama & $3.26 \mathrm{E}-23$ & $5.41 \mathrm{E}-41$ & $1.34 \mathrm{E}-80$ & $3.06 \mathrm{E}-63$ & $1.56 \mathrm{E}-47$ \\
\cline { 2 - 7 } & Std. & $1.75 \mathrm{E}-22$ & $2.91 \mathrm{E}-40$ & $5.60 \mathrm{E}-80$ & $1.65 \mathrm{E}-62$ & $8.38 \mathrm{E}-47$ \\
\hline \multirow{5}{*}{$F 7$} & En iyi & $0.00 \mathrm{E}+00$ & $0.00 \mathrm{E}+00$ & $0.00 \mathrm{E}+00$ & $0.00 \mathrm{E}+00$ & $0.00 \mathrm{E}+00$ \\
\cline { 2 - 7 } & Ortalama & $0.00 \mathrm{E}+00$ & $0.00 \mathrm{E}+00$ & $0.00 \mathrm{E}+00$ & $0.00 \mathrm{E}+00$ & $0.00 \mathrm{E}+00$ \\
\cline { 2 - 7 } & Std. & $0.00 \mathrm{E}+00$ & $0.00 \mathrm{E}+00$ & $0.00 \mathrm{E}+00$ & $0.00 \mathrm{E}+00$ & $0.00 \mathrm{E}+00$ \\
\hline \multirow{5}{*}{$F 9$} & En iyi & $\mathbf{1 . 0 0 E}+\mathbf{0 0}$ & $\mathbf{1 . 0 0 E}+\mathbf{0 0}$ & $1.90 \mathrm{E}+00$ & $3.24 \mathrm{E}+00$ & $7.28 \mathrm{E}+00$ \\
\cline { 2 - 7 } & Ortalama & $1.94 \mathrm{E}+00$ & $5.98 \mathrm{E}+00$ & $9.67 \mathrm{E}+00$ & $1.28 \mathrm{E}+01$ & $1.65 \mathrm{E}+01$ \\
\cline { 2 - 7 } & Std. & $1.46 \mathrm{E}+00$ & $3.05 \mathrm{E}+00$ & $4.58 \mathrm{E}+00$ & $4.93 \mathrm{E}+00$ & $6.66 \mathrm{E}+00$ \\
\hline \multirow{5}{*}{$F 10$} & En iyi & $1.99 \mathrm{E}-54$ & $8.70 \mathrm{E}-98$ & $8.79 \mathrm{E}-135$ & $\mathbf{0 . 0 0 E}+\mathbf{0 0}$ & $\mathbf{0 . 0 0 E}+\mathbf{0 0}$ \\
\cline { 2 - 7 } & Ortalama & $3.33 \mathrm{E}-03$ & $5.90 \mathrm{E}-70$ & $7.42 \mathrm{E}-103$ & $3.00 \mathrm{E}-144$ & $0.00 \mathrm{E}+00$ \\
\cline { 2 - 7 } & Std. & $1.79 \mathrm{E}-02$ & $2.36 \mathrm{E}-69$ & $3.97 \mathrm{E}-102$ & $1.54 \mathrm{E}-143$ & $0.00 \mathrm{E}+00$ \\
\cline { 2 - 7 } & En iyi & $-\mathbf{7 . 8 3 E}+\mathbf{0 2}$ & $-7.69 \mathrm{E}+02$ & $-7.55 \mathrm{E}+02$ & $-7.55 \mathrm{E}+02$ & $-7.69 \mathrm{E}+02$ \\
\cline { 2 - 7 } & Ortalama & $-7.46 \mathrm{E}+02$ & $-7.11 \mathrm{E}+02$ & $-7.08 \mathrm{E}+02$ & $-6.94 \mathrm{E}+02$ & $-6.91 \mathrm{E}+02$ \\
\cline { 2 - 7 } & Std. & $2.29 \mathrm{E}+01$ & $3.46 \mathrm{E}+01$ & $3.13 \mathrm{E}+01$ & $2.81 \mathrm{E}+01$ & $3.11 \mathrm{E}+01$ \\
\hline \multirow{3}{*}{$F 11$} & En iyi & $0.00 \mathrm{E}+00$ & $0.00 \mathrm{E}+00$ & $0.00 \mathrm{E}+00$ & $0.00 \mathrm{E}+00$ & $0.00 \mathrm{E}+00$ \\
\cline { 2 - 7 } & Ortalama & $0.00 \mathrm{E}+00$ & $0.00 \mathrm{E}+00$ & $0.00 \mathrm{E}+00$ & $0.00 \mathrm{E}+00$ & $0.00 \mathrm{E}+00$ \\
\cline { 2 - 7 } & Std. & $0.00 \mathrm{E}+00$ & $0.00 \mathrm{E}+00$ & $0.00 \mathrm{E}+00$ & $0.00 \mathrm{E}+00$ & $0.00 \mathrm{E}+00$ \\
\hline & En iyi & $-1.00 \mathrm{E}+00$ & $-1.00 \mathrm{E}+00$ & $-1.00 \mathrm{E}+00$ & $-1.00 \mathrm{E}+00$ & $-1.00 \mathrm{E}+00$ \\
\cline { 2 - 7 } & Ortalama & $-1.00 \mathrm{E}+00$ & $-1.00 \mathrm{E}+00$ & $-1.00 \mathrm{E}+00$ & $-1.00 \mathrm{E}+00$ & $-1.00 \mathrm{E}+00$ \\
\cline { 2 - 7 } & Std. & $0.00 \mathrm{E}+00$ & $0.00 \mathrm{E}+00$ & $0.00 \mathrm{E}+00$ & $0.00 \mathrm{E}+00$ & $0.00 \mathrm{E}+00$ \\
\hline
\end{tabular}
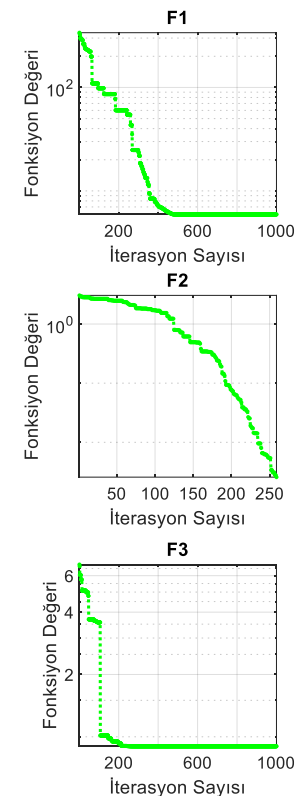

F4

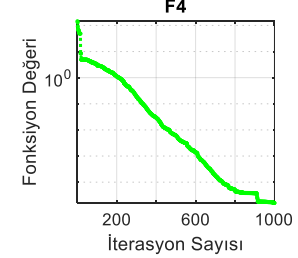

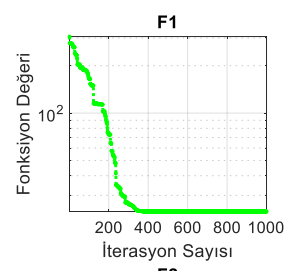
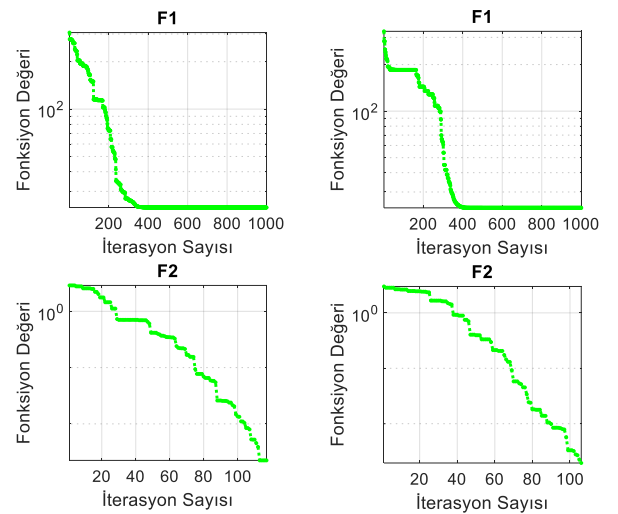

F3
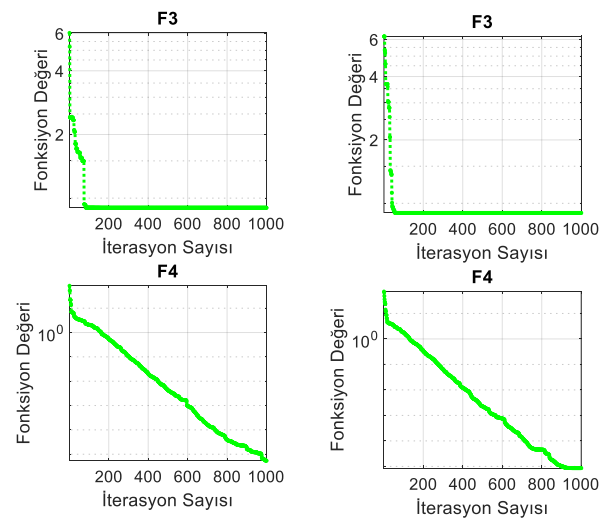

F4

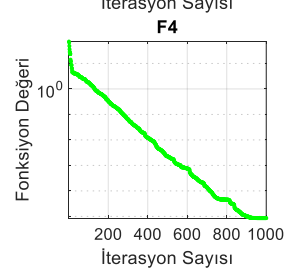

Iterasyon Sayis!
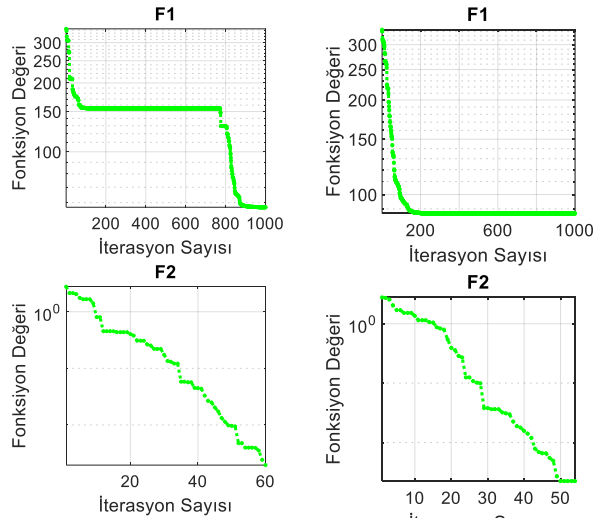

F3

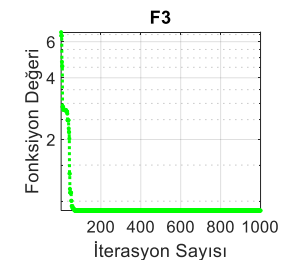

F4

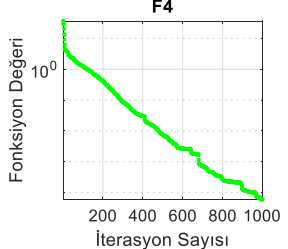

Iterasyon Sayısı
F2

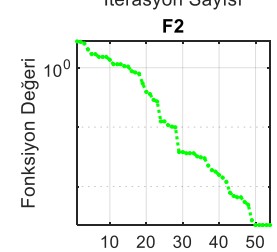

Iterasyon Sayıs।

F3

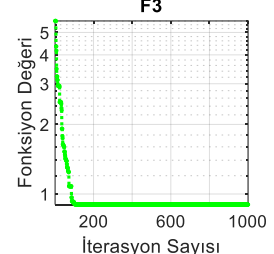

F4

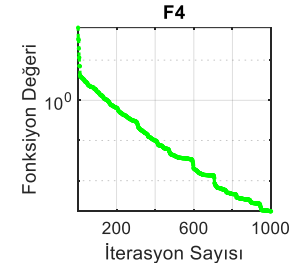



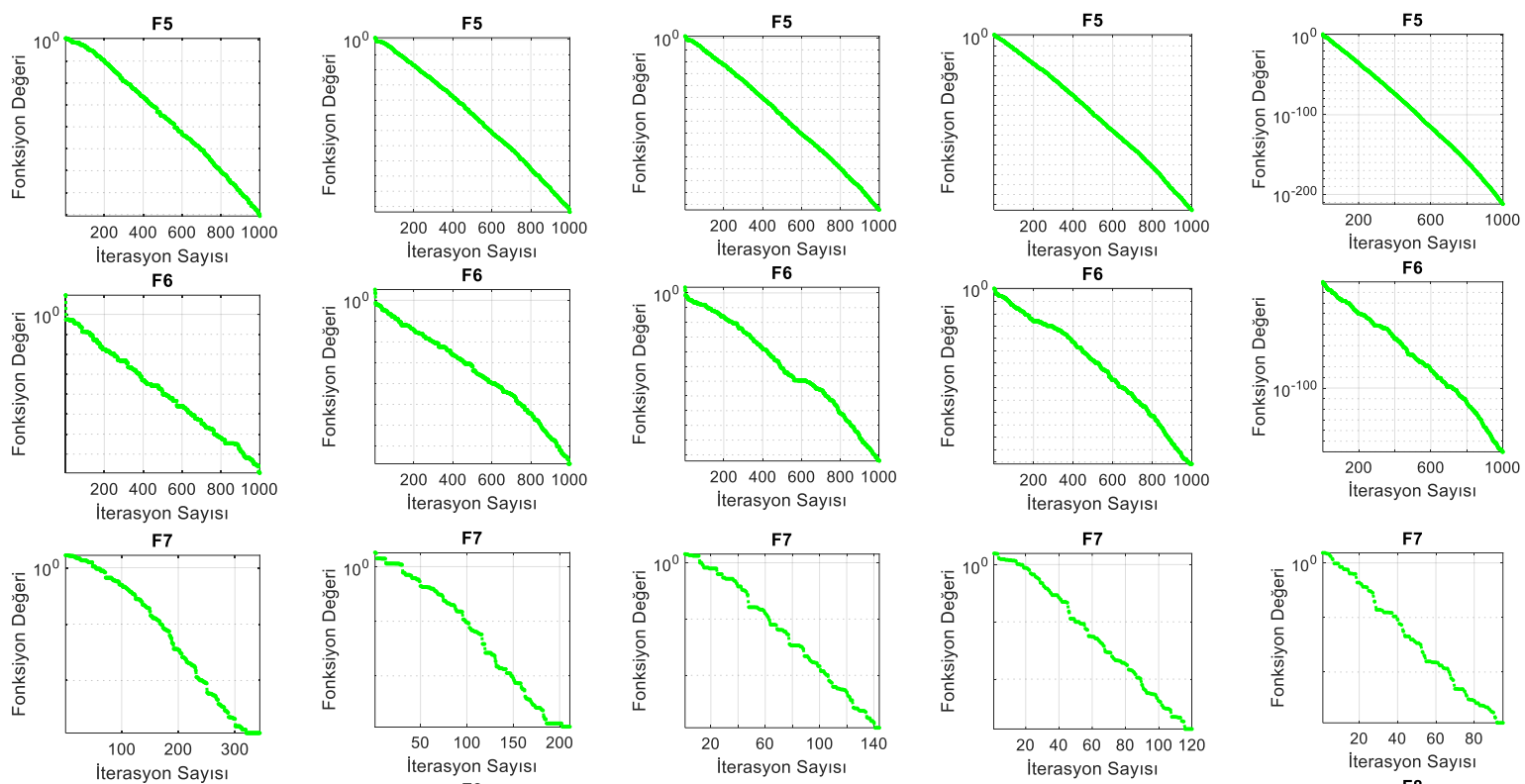

F8
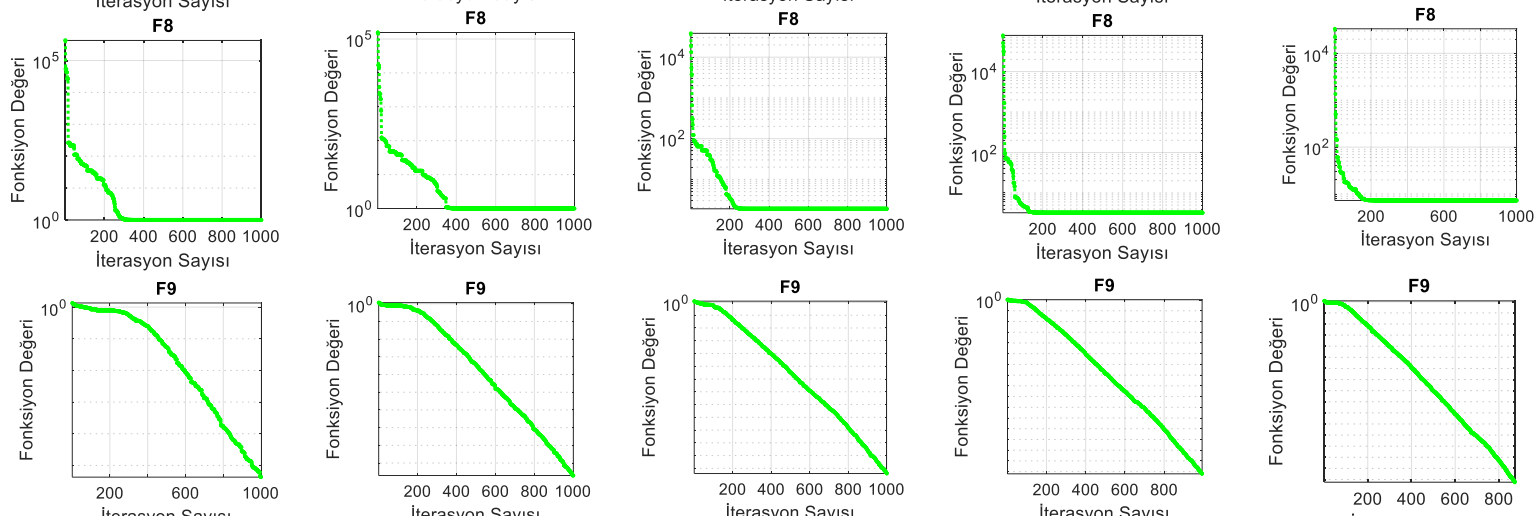

F9

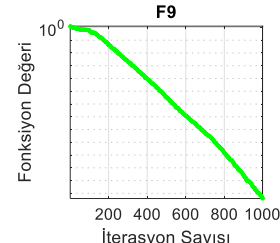

F9

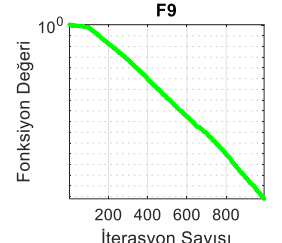

F9

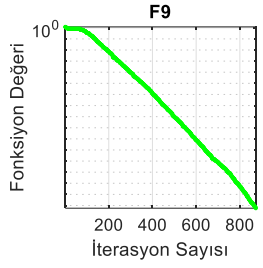

F10

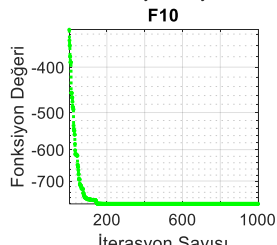

F10
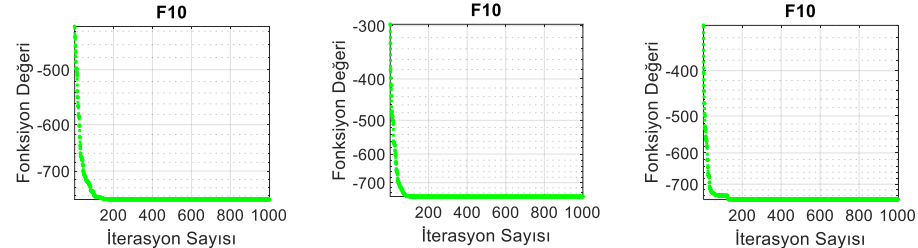

F10

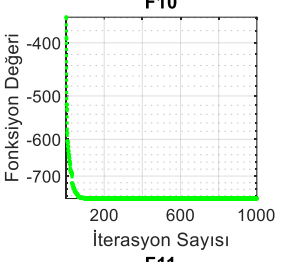

F11

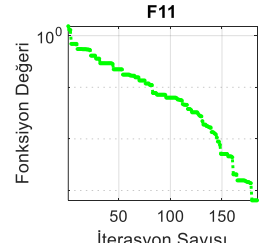

F11

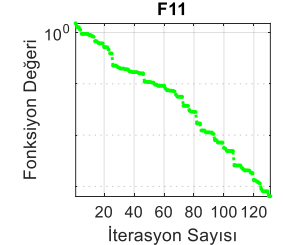

F11

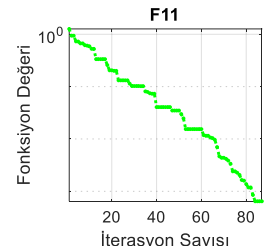

F11
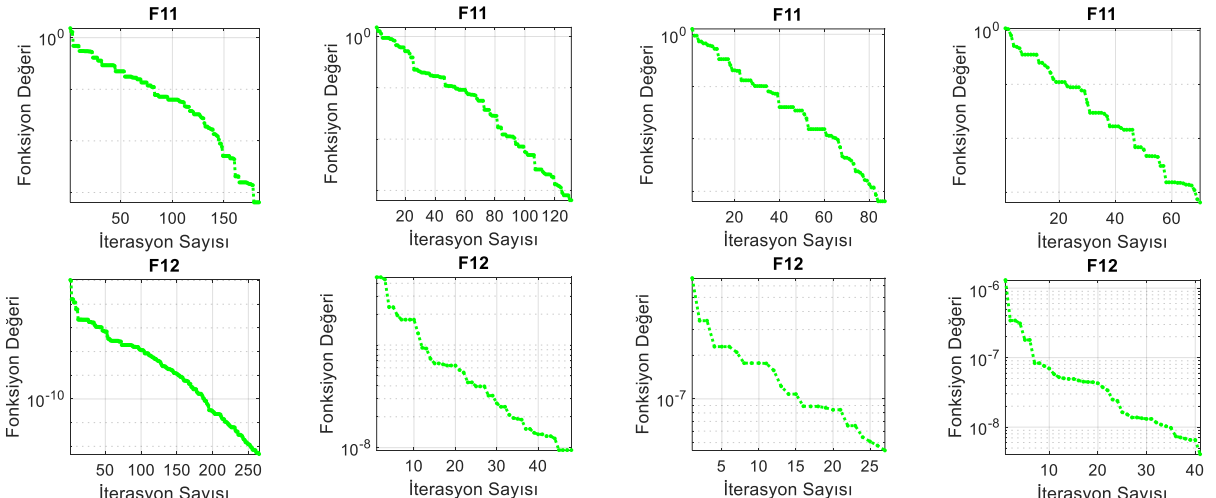

F12
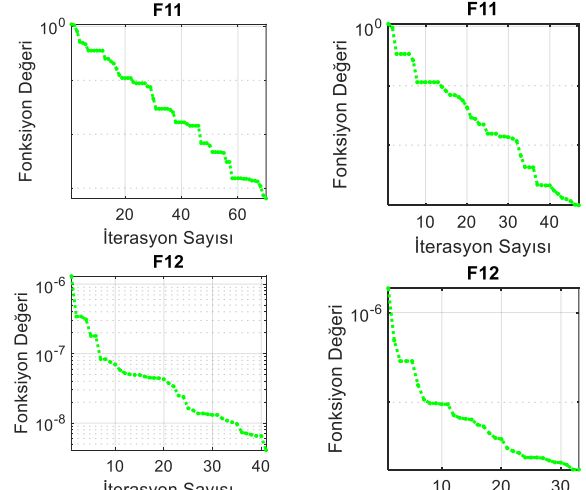

F12

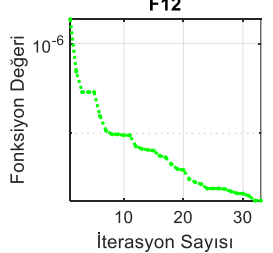

Şekil 6. Çok modlu fonksiyonlar (vektör saylsı:50)

Sonuçlar incelendiğinde GBO algoritmasının $0,1,0,3,0,5$, 0,7 ve 0,9 parametre değerleri için tek modlu fonksiyonlarda başarı elde ettiği en iyi durum parametre değeri 0,9 olduğu durumudur. Tüm parametre değerleri için vektör sayıları 10,25 ve 50 değerlerinde algoritmanın elde etiği sonuçların değişmediği görülmüştür.
Çok modlu fonksiyonlarda ise parametre değeri 0,9 olduğu durumda algoritma en iyi sonucu bulmuştur. 3 farklı vektör say değerlerinin uygulama sonucunda ise algoritma iyi sonucu elde etmek için herhangi bir farklılığın olmadığı görülmüsstür. Parametre değeri 0,9 olduğu durumda tek modlu fonksiyonlarda daha fazla en iyi sonucu elde ederken çok modlu fonksiyonlarda ise tek modlulara göre daha az en iyi sonuç elde etmiştir 


\section{Sonuçlar}

$\mathrm{Bu}$ çalışmada GBO algoritmasının parametre ayarlaması yapılmıştır. Parametrenin en iyi değerini elde edebilmek için 12 farklı tek modlu ve 12 farklı çok modlu test fonksiyonları kullanılmıştır. Aynı zamanda vektör sayısının ayarlaması da yapılmıştır. Parametre değerleri en iyi soncu en fazla hangi değerde buluyor ise o parametre değeri tavsiye niteliğinde olacaktır. Algoritmanın parametre değeri [0,1-0,9] aralığındadır. Vektör sayıs ise 10,25 ve 50 dir. GBO algoritması test fonksiyonlarına uygulandıktan sonra elde edilen sonuçlar incelendiğinde parametre değeri 0,9 olduğunda en yüksek başarı elde edilmiştir. Vektör sayısı denendiğinde ise algoritma sonuçlarında herhangi bir değişme olmamıştır. Bu sebepten dolayı GBO algoritması için 0,9 parametre değeri en iyi sonu elde etmektedir. Gelecek çalışmalarda ise GBO algoritmasının parametre değeri 0,9 alındığında daha iyi sonuçlar elde edeceği düşünüldüğünden tavsiye edilmektedir.

\section{Teșekkür}

Osmaniye Korkut Ata Üniversitesi ve Şırnak Üniversitesi bilimsel araştırma projeleri koordinatörlüklerine desteklerinden dolayı teşekkür ederiz.

\section{Kaynakça}

Aala Kalananda, V. K. R., \& Komanapalli, V. L. N. (2021). A combinatorial social group whale optimization algorithm for numerical and engineering optimization problems. Applied $\begin{array}{llll}\text { Soft Computing, } & \text { 99, }\end{array}$ doi:https://doi.org/10.1016/j.asoc.2020.106903

Ahmadianfar, I., Bozorg-Haddad, O., \& Chu, X. (2020). Gradient-based optimizer: A new metaheuristic optimization algorithm. Information Sciences, 540, 131-159. doi:https://doi.org/10.1016/j.ins.2020.06.037

Akay, B., \& Karaboga, D. (2012). A modified Artificial Bee Colony algorithm for real-parameter optimization. Information Sciences, 192, 120-142. doi:https://doi.org/10.1016/j.ins.2010.07.015

Alavidoost, M. H., Zarandi, M. H. F., Tarimoradi, M., \& Nemati, Y. (2017). Modified genetic algorithm for simple straight and U-shaped assembly line balancing with fuzzy processing times. Journal of Intelligent Manufacturing, 28(2), 313-336. doi:10.1007/s10845-014-0978-4

Beskirli, A., Beskirli, M., Hakli, H., \& Uguz, H. (2018). Comparing energy demand estimation using artificial algae algorithm: The case of Turkey. Journal of Clean Energy Technologies, 6(4),

349-352. doi:10.18178/jocet.2018.6.4.487

Beşkirli, A., \& Dağ, İ. (2020). A new binary variant with transfer functions of Harris Hawks Optimization for binary wind turbine micrositing. Energy Reports, 6, 668-673. doi:https://doi.org/10.1016/j.egyr.2020.11.154

Beşkirli, A., Özdemir, D., \& Temurtaş, H. (2020). A comparison of modified tree-seed algorithm for high-dimensional numerical functions. Neural Computing and Applications, 32(11), 6877-6911. doi:10.1007/s00521-019-04155-3

Beşkirli, A., Temurtaş, H., \& Özdemir, D. (2020). Determination with Linear Form of Turkey's Energy Demand Forecasting by the Tree Seed Algorithm and the Modified Tree Seed
Algorithm. Advances in Electrical and Computer Engineering, 20(2), 27-34. doi:10.4316/AECE.2020.02004

Dhiman, G., \& Kaur, A. (2019). STOA: A bio-inspired based optimization algorithm for industrial engineering problems. Engineering Applications of Artificial Intelligence, 82, 148174. doi:https://doi.org/10.1016/j.engappai.2019.03.021

Houssein, E. H., Saad, M. R., Hashim, F. A., Shaban, H., \& Hassaballah, M. (2020). Lévy flight distribution: A new metaheuristic algorithm for solving engineering optimization problems. Engineering Applications of Artificial Intelligence, 94, 103731 . doi:https://doi.org/10.1016/j.engappai.2020.103731

Huerta, I. I., Neira, D. A., Ortega, D. A., Varas, V., Godoy, J., \& Asín-Achá, R. (2022). Improving the state-of-the-art in the Traveling Salesman Problem: An Anytime Automatic Algorithm Selection. Expert Systems with Applications, 187, 115948. doi:https://doi.org/10.1016/j.eswa.2021.115948

Kıran, M. S., \& Findik, O. (2015). A directed artificial bee colony algorithm. Applied Soft Computing, 26, 454-462. doi:https://doi.org/10.1016/j.asoc.2014.10.020

Kutlu Onay, F., \& Aydemir, S. B. (2022). Chaotic hunger games search optimization algorithm for global optimization and engineering problems. Mathematics and Computers in Simulation, 192, 514-536. doi:https://doi.org/10.1016/j.matcom.2021.09.014

Makhloufi, S. (2015). Comparative study between classical methods and genetic algorithms for sizing remote PV systems. International Journal of Energy and Environmental Engineering, 6(3), 221-231. doi:10.1007/s40095-015-0170-4

Salgotra, R., Singh, U., Singh, G., Mittal, N., \& Gandomi, A. H. (2021). A self-adaptive hybridized differential evolution naked mole-rat algorithm for engineering optimization problems. Computer Methods in Applied Mechanics and Engineering, 383, 113916. doi:https://doi.org/10.1016/j.cma.2021.113916

Shabani, A., Asgarian, B., Salido, M., \& Asil Gharebaghi, S. (2020). Search and rescue optimization algorithm: A new optimization method for solving constrained engineering optimization problems. Expert Systems with Applications, 161 , 113698. doi:https://doi.org/10.1016/j.eswa.2020.113698

Sulaiman, M. H., Mustaffa, Z., Saari, M. M., \& Daniyal, H. (2020). Barnacles Mating Optimizer: A new bio-inspired algorithm for solving engineering optimization problems. Engineering Applications of Artificial Intelligence, 87, 103330. doi:https://doi.org/10.1016/j.engappai.2019.103330

Umam, M. S., Mustafid, M., \& Suryono, S. (2021). A hybrid genetic algorithm and tabu search for minimizing makespan in flow shop scheduling problem. Journal of King Saud University - Computer and Information Sciences. doi:https://doi.org/10.1016/j.jksuci.2021.08.025 